\title{
Stagnation-point flows with stretching surfaces: A unified formulation and new results
}

\author{
Patrick Weidman \\ Department of Mechanical Engineering \\ University of Colorado \\ Boulder, CO 80309-0427 USA \\ M. R. Turner \\ Department of Mathematics \\ University of Surrey \\ Guildford, Surrey GU2 7XH UK
}

\begin{abstract}
A unified formulation for stagnation-point flows and linearly stretching plates is given wherein the two can occur separately or in unison. Reductions to known cases are given. It is noticed that previous work on stretching plates beneath planar and axisymmetric stagnation point flows have respectively aligned planar stretching and axisymmetric stretching. The general formulation reveals other combinations of stretching beneath stagnation-point flows exist and three new cases are studied in detail. The linear stability of dual and multiple solutions are calculated.
\end{abstract}




\section{Introduction}

A unified derivation of stagnation-point flows and linearly stretching plate flows is given wherein the two can occur in unison. Reductions to cases previously studied are given. In the previous work on stretching plates beneath planar and axisymmetric stagnation point flows it is observed that the planar stretching and axisymmetric stretching are aligned with the respective flow. The general formulation considered here reveals new combinations of stretching beneath stagnation-point flows, three of which are studied in detail.

It is well-known that the solution for stagnation-point flows represent local solutions about the stagnation point; see Schlichting (1960). Nevertheless, the flow in the neighborhood of a stagnation point has great importance in that the stagnation point represents the location of highest pressure and, in thermally active flows, the highest rate heat transfer. Stagnation-point flows can be used to model the stagnation region between convection rolls. Another application is to the steady and unsteady modeling of the float height of a rotating air hockey disk; see Weidman and Sprague (2015).

The classic stagnation-point flows which may be obtained from our general formulation are the two-dimensional stagnation-point flow of Hiemenz (1911), the axisymmetric three-dimensional stagnation-point flow of Homann (1936), and the non-axisymmetric threedimensional stagnation-point flow of Howarth (1951). Plots of the similarity solutions for Hiemenz and Homann stagnation-point flows may be found in Schlichting (1960). The wall stretching problems which may be obtained are the linearly stretching surface of Crane (1970) and the bi-axial wall stretching problem of Wang (1984) which includes radial wall stretching as a special case. The general formulation is seen to also reduce to linear stretching beneath Hiemenz stagnation-point flow as studied by Mahapatra and Gupta (2002) and to radial stretching beneath Homann stagnation-point flow as studied by Mahapatra and Gupta (2003).

The three new problems studied in depth here are radial stretching of a surface beneath two-dimensional Hiemenz stagnation-point flow, linear stretching transverse to Hiemenz stagnation-point flow and linear stretching beneath axisymmetric Homann stagnation-point flow. One could also consider the more general cases of Crane stretching beneath Howarth stagnation-point flow and bi-axial Wang stretching beneath Howarth stagnation-point flow, but these multi-parameter problems are left for future study. 


\section{Problem formulation}

Cartesian coordinates $(x, y, z)$ with coordinate velocities $(u, v, w)$ are employed to describe stagnation-point flows, flows induced by linearly stretching surfaces, or a combination of the two. The far field irrotational flow is given as

$$
u(x, y, z)=a x, \quad v(x, y, z)=b y \quad(z \rightarrow \infty)
$$

where $a$ and $b$ are constant stagnation flow strain rates and the motion on the impermeable surface located at $z=0$ is

$$
u(x, y, 0)=c x, \quad v(x, y, 0)=d y, \quad w(x, y, 0)=0
$$

where $c$ and $d$ are constant stretching strain rates. A schematic diagram of the general problem is given in figure 1. Axisymmetric stagnation-point flow is obtained for $b=a$ and axisymmetric plate stretching is obtained for $d=c$.

We posit the similarity solution in the form

$$
u(x, \eta)=\alpha x F^{\prime}(\eta), \quad v(y, \eta)=\beta y G^{\prime}(\eta), \quad \eta=\sqrt{\frac{\gamma}{\nu} z}
$$

which satisfies the continuity equation for plate normal velocities if

$$
w(\eta)=-\sqrt{\frac{\nu}{\gamma}}[\alpha F(\eta)+\beta G(\eta)]
$$

where $\nu$ is the kinematic viscosity of the fluid. Inserting this similarity solution into the steady, incompressible Navier-Stokes equations yields the coupled pair of nonlinear ordinary differential equations

$$
\begin{aligned}
& \alpha \gamma F^{\prime \prime \prime}+\alpha^{2}\left(F F^{\prime \prime}-F^{2}\right)+\alpha \beta G F^{\prime \prime}+a^{2}=0 \\
& \beta \gamma G^{\prime \prime \prime}+\beta^{2}\left(G G^{\prime \prime}-G^{2}\right)+\alpha \beta F G^{\prime \prime}+b^{2}=0
\end{aligned}
$$

to be solved with plate and far-field conditions

$$
\begin{aligned}
& F(0)=0, \quad F^{\prime}(0)=\frac{c}{\alpha}, \quad F^{\prime}(\infty)=\frac{a}{\alpha} \\
& G(0)=0, \quad G^{\prime}(0)=\frac{d}{\beta}, \quad G^{\prime}(\infty)=\frac{b}{\beta} .
\end{aligned}
$$


After solving the above boundary-value problem one can compute the pressure field to be

$$
p(x, \eta)=p_{0}-\frac{\rho}{2}\left(a^{2} x^{2}+b^{2} y^{2}\right)-\frac{\rho \nu}{2 \gamma}(\alpha F+\beta G)^{2}+\rho \nu\left(\alpha F^{\prime}+\beta G^{\prime}\right)
$$

where $\rho$ is the fluid density, the wall shear stresses as

$$
\tau_{x}=\rho \alpha \sqrt{\gamma \nu} x F^{\prime \prime}(0), \quad \tau_{y}=\rho \beta \sqrt{\gamma \nu} y G^{\prime \prime}(0)
$$

and the velocity in the far field

$$
w(\infty)=-\sqrt{\frac{\nu}{\gamma}}[\alpha F(\infty)+\beta G(\infty)] .
$$

\section{Reduction to known cases}

In the following we find reductions of the above formulation to specific cases reported in the literature.

\subsection{Hiemenz stagnation-point flow}

Upon choosing $b=c=d=0$ and setting $\alpha=\gamma=a$ and $\beta=0$ one obtains the boundaryvalue problem

$$
F^{\prime \prime \prime}+F F^{\prime \prime}-F^{2}+1=0 ; \quad F(0)=0, \quad F^{\prime}(0)=0, \quad F^{\prime}(\infty)=1
$$

in which the independent variable is $\eta=\sqrt{a / \nu} z$. This is the planar stagnation-point flow problem found by Hiemenz (1911).

\subsection{Homann stagnation-point flow}

On choosing $b=a, c=d=0$ and setting $\alpha=\beta=\gamma=a$ one finds symmetrical equations for $F(\eta)$ and $G(\eta)$ with identical boundary conditions. Thus $G(\eta) \equiv F(\eta)$ and this gives rise to the single boundary-value problem

$$
F^{\prime \prime \prime}+2 F F^{\prime \prime}-F^{2}+1=0 ; \quad F(0)=0, \quad F^{\prime}(0)=0, \quad F^{\prime}(\infty)=1
$$

where again the independent variable is $\eta=\sqrt{a / \nu} z$. This is the axisymmetric stagnationpoint flow found by Homann (1936). 


\subsection{Howarth stagnation-point flow}

Here we choose $c=d=0$ and set $\alpha=a, \beta=b$ and $\gamma=a$ to obtain the coupled equations

$$
\begin{gathered}
F^{\prime \prime \prime}+F F^{\prime \prime}-F^{2}+\sigma G F^{\prime \prime}+1=0 \\
\frac{1}{\sigma} G^{\prime \prime \prime}+G G^{\prime \prime}-G^{2}+\frac{1}{\sigma} F G^{\prime \prime}+1=0
\end{gathered}
$$

in which $\sigma=b / a$. These equations are to be solved with boundary and far-field conditions

$$
F(0)=G(0)=0, \quad F^{\prime}(0)=G^{\prime}(0)=0, \quad F^{\prime}(\infty)=G^{\prime}(\infty)=1
$$

where again the independent variable is $\eta=\sqrt{a / \nu} z$. This is recognized as the "orthogonal Hiemenz" stagnation-point flow problem reported by Howarth (1951). Howarth solved the nodal-point system for selected values in the range $0 \leq \sigma \leq 1$ and Davey (1961) solved the saddle-point system for selected values in the range $-1 \leq \sigma \leq 0$.

\subsection{Crane stretching plate flow}

Here we set $a=b=d=0$ and choose $\beta=0$ with $\alpha=\gamma=c$ to obtain the boundary-value problem

$$
F^{\prime \prime \prime}+F F^{\prime \prime}-F^{2}=0 ; \quad F(0)=0, \quad F^{\prime}(0)=1, \quad F^{\prime}(\infty)=0
$$

where now the independent variable is $\eta=\sqrt{c / \nu} z$. This is recognized as the planar plate stretching problem attributed to Crane (1970). Recently, Weidman and Ishak (2015) considered Crane's shrinking plate problem and found a dual solution. The interesting feature of the dual solution is that it decays algebraically in the far field.

\subsection{Wang stretching plate flow}

Upon choosing $a=b=0$ and setting $\alpha=\beta=\gamma=c$ one finds the coupled system of equations

$$
F^{\prime \prime \prime}+(F+G) F^{\prime \prime}-F^{\prime 2}=0 ; \quad G^{\prime \prime \prime}+(F+G) G^{\prime \prime}-G^{2}=0
$$

to be solved with boundary and far-field conditions

$$
F(0)=0, \quad F^{\prime}(0)=1, \quad F^{\prime}(\infty)=0 ; \quad G(0)=0, \quad G^{\prime}(0)=\sigma, \quad G^{\prime}(\infty)=0
$$

where $\sigma=d / c$ and the plate-normal coordinate is $\eta=\sqrt{c / \nu} z$. This orthogonal plate stretching system of equations was found by Wang (1984) who recognized that for $\sigma=1$ one has $G(\eta) \equiv F(\eta)$ which reduces the above system to

$$
F^{\prime \prime \prime}+2 F F^{\prime \prime}-F^{2}=0 ; \quad F(0)=0, \quad F^{\prime}(0)=1, \quad F^{\prime}(\infty)=0
$$


corresponding to a sheet stretching radially at strain rate $c$. Wang only considered solutions for $\sigma \geq 0$. Recently, Weidman and Ishack (2015) considered shrinking plates and found dual solutions up to a turning point $\sigma_{t}=-0.2514$.

\subsection{Crane stretching aligned with Hiemenz stagnation-point flow}

Here we choose $b=0, d=c$ and set $\beta=0$ with $\alpha=\gamma=c$ to obtain the boundary-value problem

$$
F^{\prime \prime \prime}+F F^{\prime \prime}-F^{2}+\sigma^{2}=0 ; \quad F(0)=0, \quad F^{\prime}(0)=1, \quad F^{\prime}(\infty)=\sigma
$$

where $\sigma=a / c$ and the independent variable is $\eta=\sqrt{c / \nu} z$. This system was found by Mahapatra and Gupta (2002) and solved for selected positive values of $\sigma$. Wang (2008) extended this work to shrinking surfaces.

\subsection{Radial stretching aligned with Homann stagnation-point flow}

Here we set $b=a, d=c$ and set $\alpha=\beta=\gamma=c$ to obtain the boundary-value problem

$$
F^{\prime \prime \prime}+2 F F^{\prime \prime}-F^{2}+\sigma^{2}=0 ; \quad F(0)=0, \quad F^{\prime}(0)=1, \quad F^{\prime}(\infty)=\sigma
$$

where again $\sigma=a / c$ and $\eta=\sqrt{c / \nu} z$ is the independent variable and $G(\eta) \equiv F(\eta)$. This system was found by Mahapatra and Gupta (2003) and solved for selected positive values of $\sigma$. Wang (2008) extended this work to shrinking surfaces.

We now consider three problems for stretching surfaces below stagnation-point flows not previously reported in the literature. It is noted that Mahapatra and Gupta (2002) considered planar stretching parallel to flow streamlines for the Hiemenz stagnation-point flow. Also, Mahapatra and Gupta (2003) considered radial stretching parallel to flow streamlines for the Homann stagnation-point flow. In the following we consider other orientations of wall stretching beneath these two stagnation-point flows.

In the results presented in the proceeding sections the resulting ordinary differential equations are solved using a shooting method. This method incorporates $4^{\text {th }}$ order RungeKutta integration to integrate out to $\eta_{\max }=20$, and Newton iterations to update the initial guesses for $F^{\prime \prime}(0)$ and $G^{\prime \prime}(0)$; see Press, et al (1989). The integration domain $\left[0, \eta_{\max }\right]$ and Runge-Kutta step size are varied to ensure that the results presented here are independent of these parameters. 


\section{Radial stretching beneath Hiemenz stagnation flow}

Here we take $b=0, d=c$ and set $\alpha=\beta=\gamma=a$ which gives rise to the coupled equations

$$
\begin{gathered}
F^{\prime \prime \prime}+(F+G) F^{\prime \prime}-F^{2}+1=0 \\
G^{\prime \prime \prime}+(F+G) G^{\prime \prime}-G^{2}=0
\end{gathered}
$$

for the independent variable $\eta=\sqrt{a / \nu} z$. These equations are to be solved with boundary and far-field conditions

$$
F(0)=0 \quad F^{\prime}(0)=\sigma, \quad F^{\prime}(\infty)=1 ; \quad G(0)=0 \quad G^{\prime}(0)=\sigma, \quad G^{\prime}(\infty)=0
$$

in which $\sigma=c / a$. In this case the expression for the $x$ (longitudinal) and $y$ (transverse) wall shear stresses are

$$
\tau_{x}=\rho a^{3 / 2} \nu^{1 / 2} x F^{\prime \prime}(0), \quad \tau_{y}=\rho a^{3 / 2} \nu^{1 / 2} y G^{\prime \prime}(0) .
$$

\subsection{Local analysis near $\sigma=1$}

Numerical solutions of (4.1) given in $\S 4.3$ following the primary solution branch to negative values of $\sigma$ reveals a turning point at $\sigma_{t}=-0.3737$ and following the solutions around this turning point gives a dual solution branch.

Here present a local analysis of these dual solutions at $\sigma=1$ for which the plate stretches at the same rate as the outer Hiemenz stagnation-point flow. Hence the solution for the longitudinal flow is simply $F(\eta)=F_{0}(\eta)=\eta$ and in this case we look for local solutions in the form

$$
F(\eta)=\eta+\epsilon F_{1}(\eta)+\cdots, \quad G(\eta)=G_{0}(\eta)+\epsilon G_{1}(\eta)+\cdots
$$

for $\epsilon=\sigma-1 \ll 1$. Inserting this into boundary-value problem (4.6) gives at $\mathrm{O}(1)$

$$
G_{0}^{\prime \prime \prime}+\left(\eta+G_{0}\right) G_{0}^{\prime \prime}-G_{0}^{\prime 2}=0, \quad G_{0}(0)=0, \quad G_{0}^{\prime}(0)=1, \quad G_{0}^{\prime}(\infty)=0
$$

and at $\mathrm{O}(\epsilon)$ one finds

$$
F_{1}^{\prime \prime \prime}+\left(\eta+G_{1}\right) F_{1}^{\prime \prime}-2 F_{1}^{\prime}=0, \quad F_{1}(0)=0, \quad F_{1}^{\prime}(0)=1, \quad F_{1}^{\prime}(\infty)=0
$$

and

$$
G_{1}^{\prime \prime \prime}+\left(\eta+G_{0}\right) G_{1}^{\prime \prime}+\left(F_{1}+G_{1}\right) G_{0}^{\prime \prime}-2 G_{0}^{\prime} G_{1}^{\prime}=0, \quad G_{1}(0)=0, \quad G_{1}^{\prime}(0)=1, \quad G_{1}^{\prime}(\infty)=0 .
$$


Solving this system for the upper branch solution furnishes the leading order expressions for the wall shear stress parameters

$$
F^{\prime \prime}(0)=-1.720791(\sigma-1), \quad G^{\prime \prime}(0)=-1.277071-1.803351(\sigma-1)
$$

and for the lower branch solution we find

$$
F^{\prime \prime}(0)=-1.652865(\sigma-1), \quad G^{\prime \prime}(0)=-2.496922-2.366740(\sigma-1)
$$

\subsection{Large $\sigma$ asymptotics}

The goal in this section is to find a two-term large- $\sigma$ asymptotic formulae for the wall shear stresses along the upper branch solution. This is accomplished by matching inner and outer solutions.

Inspection of Eqs. (4.1) for $\sigma \gg 1$ suggests the boundary-layer scaling

$$
\eta=\sigma^{-\alpha} \xi, \quad F(\eta)=\sigma^{\alpha} f(\xi), \quad G(\eta)=\sigma^{\alpha} g(\xi)
$$

which yields

$$
\sigma^{4 \alpha}\left(f^{\prime \prime \prime}+(f+g) f^{\prime \prime}-f^{\prime 2}\right)+1=0, \quad f(0)=0, \quad f^{\prime}(0)=\sigma^{1-2 \alpha}, \quad f^{\prime}(\infty)=\sigma^{-2 \alpha}
$$

and

$$
g^{\prime \prime \prime}+(f+g) g^{\prime \prime}-g^{\prime 2}=0, \quad g(0)=0, \quad g^{\prime}(0)=\sigma^{1-2 \alpha}, \quad g^{\prime}(\infty)=0
$$

where a prime now denotes differentiation with respect to $\xi$. We set $\alpha=1 / 2$ to maintain velocities of order unity, viz. $f^{\prime}(0)=g^{\prime}(0)=1$. This suggests the first expansion terms

$$
F(\eta)=\sigma^{1 / 2} f_{0}(\xi)+\cdots, \quad G(\eta)=\sigma^{1 / 2} g_{0}(\xi)+\cdots
$$

To ascertain the next term in the expansion we seek an outer solution of $F(\eta)$ for $\eta=\mathrm{O}(1)$ to find

$$
F(\eta)=\eta+C(\sigma)
$$

where $C$ is a constant to be determined. Matching to the leading-order inner solution (4.10) leads to

$$
C(\sigma)=f_{0 \infty} \sigma^{1 / 2}
$$

where $f_{0}(\xi) \rightarrow f_{0_{\infty}}$ as $\xi \rightarrow \infty$. Consequently, as $\eta \rightarrow 0$ we have the development

$$
F=f_{0 \infty} \sigma^{1 / 2}+\xi \sigma^{-1 / 2}
$$


which therefore requires that the expansion for $F(\eta)$ and $G(\eta)$ be of the form

$$
\begin{gathered}
F(\eta)=\sigma^{1 / 2} f_{0}(\xi)+\sigma^{-1 / 2} f_{1}(\xi)+\cdots \\
G(\eta)=\sigma^{1 / 2} g_{0}(\xi)+\sigma^{-1 / 2} g_{1}(\xi)+\cdots
\end{gathered}
$$

Inserting (4.13) into boundary-value problem (4.1) gives at $O\left(\sigma^{1 / 2}\right)$

$$
f_{0}^{\prime \prime \prime}+2 f_{0} f_{0}^{\prime \prime}-f_{0}^{\prime 2}=0, \quad f_{0}(0)=0, \quad f_{0}^{\prime}(0)=1, \quad f_{0}^{\prime}(\infty)=0
$$

whereby $g_{0}(\xi) \equiv f_{0}(\xi)$ which furnishes the next order system at $O\left(\sigma^{-1 / 2}\right)$

$$
\begin{aligned}
& f_{1}^{\prime \prime \prime}+2 f_{0} f_{1}^{\prime \prime}+\left(f_{1}+g_{1}\right) f_{0}^{\prime \prime}-2 f_{0}^{\prime} f_{1}^{\prime}=0, \quad f_{1}(0)=0, \quad f_{1}^{\prime}(0)=0, \quad f_{1}^{\prime}(\infty)=1 \\
& g_{1}^{\prime \prime \prime}+2 f_{0} g_{1}^{\prime \prime}+\left(f_{1}+g_{1}\right) f_{0}^{\prime \prime}-2 f_{0}^{\prime} g_{1}^{\prime}=0, \quad g_{1}(0)=0, \quad g_{1}^{\prime}(0)=0, \quad g_{1}^{\prime}(\infty)=0 .
\end{aligned}
$$

Solving the above equations leads to the two-term asymptotic behaviors

$$
\begin{aligned}
& F^{\prime \prime}(0) \sim-1.173721 \sigma^{3 / 2}+0.452212 \sigma^{1 / 2} \\
& G^{\prime \prime}(0) \sim-1.173721 \sigma^{3 / 2}-0.076447 \sigma^{1 / 2}
\end{aligned}
$$

\subsection{Presentation of results}

Numerical integrations for the longitudinal and transverse shear stress parameters $F^{\prime \prime}(0)$ and $G^{\prime \prime}(0)$ as a function of $\sigma$ are shown in figures $2 \mathrm{a}$ and $2 \mathrm{~b}$, respectively. A turning point $\sigma_{t}=$ -0.3737 reveals that dual solutions exist for $\sigma>\sigma_{t}$ and no solutions are found for $\sigma<\sigma_{t}$. The insets in figures 2a,b show details of the shear stress parameters in the neighborhood of the turning point. Note the focal point in $F^{\prime \prime}(0)$ at $\sigma=1$ where the primary solution and dual solution branches cross.

In figures 2a,b the leading-order local solutions for the primary branch at $\sigma=1$ are shown as the dashed lines and those for the dual solution branch are shown as the dotted lines. Figure 2c shows a comparison of large- $\sigma$ asymptotic behaviors (4.15) for longitudinal and transverse wall shear stresses for the primary branch with corresponding numerical results. The dashed line is the one-term asymptotic behavior for both $F^{\prime \prime}(0)$ and $G^{\prime \prime}(0)$ and the dotted lines are the two-term asymptotic behaviors. The two-term result for $F^{\prime \prime}(0)$ is seen to approach the primary solution branch at large $\sigma$ and the two-term result for $G^{\prime \prime}(0)$ is not visible since it precisely overlays the primary solution branch.

Sample longitudinal and transverse velocity profiles are displayed in figure $2 \mathrm{~d}$. The profiles are shown at the turning point $\sigma_{t}=-0.3737$ and at $\sigma=\{0,1,2,3\}$ with primary 
branch solutions displayed as solid lines, dual branch solutions displayed as dashed lines, and turning point solutions shown as the dash-dot-dash lines. The profiles of $G^{\prime}(\eta)$ for the dual branch solutions with $\sigma>0$ have a region of reverse flow away from the plate, while in the primary branch solutions $G^{\prime}(\eta)>0$ for all $\eta \in\left[0, \eta_{\max }\right]$.

\subsection{Stability of Dual Solutions}

Introducing the dimensionless time $\tau=$ at we now include the unsteady acceleration in the Navier-Stokes equations which gives rise to the coupled nonlinear system of equations

$$
\begin{gathered}
F^{\prime \prime \prime}+(F+G) F^{\prime \prime}-F^{2}-F_{\tau}^{\prime}+1=0 \\
G^{\prime \prime \prime}+(F+G) G^{\prime \prime}-G^{2}-G_{\tau}^{\prime}=0 .
\end{gathered}
$$

To study the stability of these self-similar system of equations, we follow Merkin (1985) and write

$$
F(\eta, \tau)=F_{0}(\eta)+\delta e^{-\lambda \tau} F_{1}(\eta), \quad G(\eta, \tau)=G_{0}(\eta)+\delta e^{-\lambda \tau} G_{1}(\eta)
$$

where $\lambda$ is an eigenvalue determining the linear stability of the flow and $F_{0}$ and $G_{0}$ are solutions of the steady problem. Assuming that $\delta \ll 1$, insertion of (4.17) into (4.16) and linearizing yields

$$
\begin{aligned}
& F_{1}^{\prime \prime \prime}+\left(F_{0}+G_{0}\right) F_{1}^{\prime \prime}+F_{0}^{\prime \prime}\left(F_{1}+G_{1}\right)-2 F_{0}^{\prime} F_{1}^{\prime}+\lambda F_{1}^{\prime}=0 \\
& G_{1}^{\prime \prime \prime}+\left(F_{0}+G_{0}\right) G_{1}^{\prime \prime}+G_{0}^{\prime \prime}\left(F_{1}+G_{1}\right)-2 G_{0}^{\prime} G_{1}^{\prime}+\lambda G_{1}^{\prime}=0 .
\end{aligned}
$$

Solutions of this system with homogeneous boundary conditions

$$
F_{1}(0)=F_{1}^{\prime}(0)=F_{1}^{\prime}(\infty)=0, \quad G_{1}(0)=G_{1}^{\prime}(0)=G_{1}^{\prime}(\infty)=0
$$

yields a sequence of eigenvalues $\lambda_{1}<\lambda_{2}<\lambda_{3}<\cdots$ and the lowest eigenvalue, $\lambda_{1}$, determines the stability. Unlike Merkin (1985) we now have a coupled system of equations. In this case we take the homogeneous boundary conditions (4.19a) along with the choice

$$
F_{1}^{\prime \prime}(0)=1
$$

and solve the above system by shooting from $\eta=0$ to $\eta=\eta_{\max }$ and updating the values of $\lambda_{1}$ and $G_{1}^{\prime \prime}(0)$ via Newton iterations, using the same numerical approach developed to solve the steady problem. The results of the shooting method for calculating $\lambda_{1}$ were checked against 
those using a global eigenvalue solution approach (Schmid \& Henningson, 2012) which finds all the eigenvalues at once.

Results of a comprehensive study yields the $\sigma$-variation of the smallest eigenvalue as plotted in figure $2 \mathrm{e}$ and the associated values of $G_{1}^{\prime \prime}(0)$ plotted in figure $2 \mathrm{f}$. It is clear from figure $2 \mathrm{e}$ that the primary solutions are stable while the dual solutions are unstable. The blow-up of $G_{1}^{\prime \prime}(0)$ at $\sigma=1$ is associated with the solution $F_{1}^{\prime \prime}(\eta)=0$ with $G^{\prime \prime}(0)$ finite, for that value of $\sigma$.

\section{Crane stretching transverse to Hiemenz stagnation flow}

In this case we take $b=c=0$ and set $\alpha=\beta=\gamma=a$ which gives rise to the coupled nonlinear equations

$$
\begin{gathered}
F^{\prime \prime \prime}+(F+G) F^{\prime \prime}-F^{2}+1=0 \\
G^{\prime \prime \prime}+(F+G) G^{\prime \prime}-G^{2}=0
\end{gathered}
$$

in which the independent variable is $\eta=\sqrt{a / \nu} z$. These equations are to be solved with boundary and far-field conditions

$$
F(0)=0 \quad F^{\prime}(0)=0, \quad F^{\prime}(\infty)=1 ; \quad G(0)=0 \quad G^{\prime}(0)=\sigma, \quad G^{\prime}(\infty)=0
$$

where now $\sigma=d / a$. The wall shear stresses are those given in Eq. (4.2).

\subsection{Local analysis near $\sigma=0$}

At $\sigma=0$ one has pure Hiemenz flow with no transverse plate stretching. In this case we look for local solutions in the form

$$
F(\eta)=F_{0}(\eta)+\sigma F_{1}(\eta)+\cdots, \quad G(\eta)=\sigma G_{1}(\eta)+\cdots
$$

for $\sigma$ small. Inserting these expansions into boundary-value problem (4.1) gives at $\mathrm{O}(1)$ the Hiemenz stagnation-point flow problem

$$
F_{0}^{\prime \prime \prime}+F_{0} F_{0}^{\prime \prime}-F_{0}^{\prime 2}+1=0, \quad F_{0}(0)=0, \quad F^{\prime}(0)=0, \quad F^{\prime}(\infty)=1
$$

and at $\mathrm{O}(\sigma)$ one finds

$$
F_{1}^{\prime \prime \prime}+\left(F_{1}+G_{1}\right) F_{0}^{\prime \prime}+F_{0} F_{1}^{\prime \prime}-2 F_{0}^{\prime} F_{1}^{\prime}=0, \quad F_{1}(0)=0, \quad F_{1}^{\prime}(0)=0, \quad F_{1}^{\prime}(\infty)=0
$$


and

$$
G_{1}^{\prime \prime \prime}+F_{0} G_{1}^{\prime \prime}=0, \quad G_{1}(0)=0, \quad G_{1}^{\prime}(0)=1, \quad G_{1}^{\prime}(\infty)=0 .
$$

Solving this system leads the the following leading-order expressions for the wall shear stress parameters,

$$
F^{\prime \prime}(0)=1.232588+0.18533 \sigma, \quad G^{\prime \prime}(0)=-0.570465 \sigma .
$$

\subsection{Large $\sigma$ asymptotics}

Analysis of the asymptotic behavior of problem (5.1) follows that given in $\S 4.2$ the only difference being that the boundary-values displayed in Eqs. (4.8) for $\alpha=1 / 2$ are now

$$
f(0)=0, \quad f^{\prime}(0)=0, \quad f^{\prime}(\infty)=\sigma^{-1}, \quad g(0)=0, \quad g^{\prime}(0)=1, \quad g^{\prime}(\infty)=0 .
$$

Matching inner boundary-layer flow with the outer flow again shows that $F(\eta)$ and $G(\eta)$ have the developments given by (4.14) and inserting this into (5.1) gives at $O(\sigma)$

$$
\begin{gathered}
f_{0}^{\prime \prime \prime}+\left(f_{0}+g_{0}\right) f_{0}^{\prime \prime}-f_{0}^{\prime 2}=0, \quad f_{0}(0)=0, \quad f_{0}^{\prime}(0)=0, \quad f_{0}^{\prime}(\infty)=0 \\
g_{0}^{\prime \prime \prime}+\left(f_{0}+g_{0}\right) g_{0}^{\prime \prime}-g_{0}^{\prime 2}=0, \quad g_{0}(0)=0, \quad g_{0}^{\prime}(0)=1, \quad g_{0}^{\prime}(\infty)=0
\end{gathered}
$$

and at $O\left(\sigma^{-1}\right)$ one finds

$$
\begin{aligned}
& f_{1}^{\prime \prime \prime}+\left(f_{0}+g_{0}\right) f_{1}^{\prime \prime}+\left(f_{1}+g_{1}\right) f_{0}^{\prime \prime}-2 f_{0}^{\prime} f_{1}^{\prime}=0, \quad f_{1}(0)=0, \quad f_{1}^{\prime}(0)=0, \quad f_{1}^{\prime}(\infty)=1 \\
& g_{1}^{\prime \prime \prime}+\left(f_{0}+g_{0}\right) g_{1}^{\prime \prime}+\left(f_{1}+g_{1}\right) f_{0}^{\prime \prime}-2 g_{0}^{\prime} g_{1}^{\prime}=0, \quad g_{1}(0)=0, \quad g_{1}^{\prime}(0)=0, \quad g_{1}^{\prime}(\infty)=0 .
\end{aligned}
$$

Solution of $(5.7)$ gives $f_{0}(\xi) \equiv 0$ and solution of the full set leads to the following two-term asymptotic behaviors

$$
\begin{aligned}
F^{\prime \prime}(0) & \sim 0.582019 \sigma^{1 / 2}, \\
G^{\prime \prime}(0) & \sim-1.0 \sigma^{3 / 2}-0.1067004 \sigma^{1 / 2} .
\end{aligned}
$$

\subsection{Presentation of results}

Results of numerical integrations for the longitudinal and transverse shear stress parameters $F^{\prime \prime}(0)$ and $G^{\prime \prime}(0)$ as a function of $\sigma$ are shown in figures 3a and 3b, respectively. A turning point at $\sigma_{t}=-0.4690$ appears giving dual solutions for $\sigma>\sigma_{t}$ and no solutions for $\sigma<\sigma_{t}$. The insets in figures $3 \mathrm{a}$,b show details of the shear stress parameters in the neighborhood of the turning point. The dashed lines in each figure are the local behaviors of the shear stress

parameters at $\sigma=0$ on the primary (upper) solution branch given in Eq. (5.5). 
Sample longitudinal and transverse velocity profiles are displayed in figure $3 \mathrm{~d}$. The profiles are shown at the turning point $\sigma_{t}=-0.4690$ and at $\sigma=\{0,1,2,3\}$ with primary branch solutions displayed as solid lines, dual branch solutions displayed as dashed lines, and turning point solutions shown as the dash-dot-dash lines. These profiles show that $G^{\prime}(\eta)$ displays similar reverse flow behavior as in $\S 4$, for $\sigma>0$.

\subsection{Stability of Dual Solutions}

As in $§ 4.4$ we introduce the dimensionless time $\tau=a t$ and include the unsteady acceleration in the Navier-Stokes equations which leads to the same coupled system of equations (4.16), which in turn leads to the eigenvalue problem governed by (4.18) with boundary conditions (4.19). It should be noted that although the eigenvalue problem is the same as for radial stretching beneath Hiemenz stagnation-point flow, the eigenvalues are different owing to the different base flow solutions $F_{0}(\eta)$ and $G_{0}(\eta)$ in Eq. (4.17).

The lowest eigenvalue for this flow are plotted in figure $3 \mathrm{e}$ with the associated value of $G_{1}^{\prime \prime}(0)$ plotted in figure 3f. Again it is clear from figure 3e that primary solutions are stable while dual solutions are unstable.

\section{Crane stretching beneath Homann stagnation flow}

We now take $b=a, d=0$ and again set $\alpha=\beta=\gamma=a$ which gives rise to the coupled equations

$$
\begin{aligned}
& F^{\prime \prime \prime}+(F+G) F^{\prime \prime}-F^{2}+1=0, \\
& G^{\prime \prime \prime}+(F+G) G^{\prime \prime}-G^{2}+1=0
\end{aligned}
$$

in which the independent variable is $\eta=\sqrt{a / \nu} z$. The relevant boundary and far-field conditions are

$$
F(0)=0 \quad F^{\prime}(0)=0, \quad F^{\prime}(\infty)=1 ; \quad G(0)=0 \quad G^{\prime}(0)=\sigma, \quad G^{\prime}(\infty)=1
$$

in which $\sigma=d / a$. Again the wall shear stresses are those given in Eq. (4.2).

\subsection{Local analysis near $\sigma=0$}

At $\sigma=0$ one has identical equations and boundary conditions giving $G(\eta) \equiv F(\eta)$. Here we seek local solutions in the form

$$
F(\eta)=F_{0}(\eta)+\sigma F_{1}(\eta)+\cdots, \quad G(\eta)=G_{0}(\eta)+\sigma G_{1}(\eta)+\cdots
$$


for $\sigma$ small. Inserting these expansions into boundary-value problem (6.1) gives at $\mathrm{O}(1)$ the Homann stagnation-point flow problem

$$
F_{0}^{\prime \prime \prime}+2 F_{0} F_{0}^{\prime \prime}-F_{0}^{\prime 2}+1=0, \quad F_{0}(0)=0, \quad F_{0}^{\prime}(0)=0, \quad F_{0}^{\prime}(\infty)=1
$$

for which $G_{0}(\eta) \equiv F_{0}(\eta)$. At $\mathrm{O}(\sigma)$ one finds

$$
F_{1}^{\prime \prime \prime}+\left(F_{0}+G_{0}\right) F_{1}^{\prime \prime}+\left(F_{1}+G_{1}\right) f_{0}^{\prime \prime}-2 F_{0}^{\prime} F_{1}^{\prime}=0, \quad F_{1}(0)=0, \quad F_{1}^{\prime}(0)=0, \quad F_{1}^{\prime}(\infty)=0
$$

and

$G_{1}^{\prime \prime \prime}+\left(F_{0}+G_{0}\right) G_{1}^{\prime \prime}+\left(F_{1}+G_{1}\right) G_{0}^{\prime \prime}-2 G_{0}^{\prime} G_{1}^{\prime}=0, \quad G_{1}(0)=0, \quad G_{1}^{\prime}(0)=1, \quad G_{1}^{\prime}(\infty)=0$.

Solving this system provides the leading order expressions for the wall shear stress parameters on Branch I as

$$
F^{\prime \prime}(0)=1.311938+0.156219 \sigma, \quad G^{\prime \prime}(0)=1.311938-0.918451 \sigma .
$$

\subsection{Large $\sigma$ asymptotics}

Analysis of the asymptotic behavior of problem (6.1) follows that given in $\S 4.2$, the only difference being that the boundary-values displayed in Eqs. (4.8) for $\alpha=1 / 2$ are now

$$
f(0)=0, \quad f^{\prime}(0)=0, \quad f^{\prime}(\infty)=\sigma^{-1}, \quad g(0)=0, \quad g^{\prime}(0)=1, \quad g^{\prime}(\infty)=\sigma^{-1} .
$$

The resulting asymptotics are seen to be identical to those given in Eq. (5.8), viz.

$$
\begin{aligned}
& F^{\prime \prime}(0) \sim 0.582019 \sigma^{1 / 2} \\
& G^{\prime \prime}(0) \sim-1.0 \sigma^{3 / 2}-0.1067004 \sigma^{1 / 2}
\end{aligned}
$$

On Branch I. Hence the unity terms in Eq. (6.1a,b) do not affect the asymptotic behavior of the system to $\mathrm{O}\left(\sigma^{1 / 2}\right)$. However, they of course do affect the full numerical solution of the problem.

\subsection{Presentation of results}

Numerical integrations provide the longitudinal and transverse shear stress parameters $F^{\prime \prime}(0)$ and $G^{\prime \prime}(0)$ as a function of $\sigma$ as shown in figures $4 \mathrm{a}$ and $4 \mathrm{~b}$, respectively. As shown in the insets, we find solutions starting on the primary branch winding down about the point 
$\left\{\sigma, F^{\prime \prime}(0)\right\}=\{-1,1\}$ for figure 4 a and the point $\left\{\sigma, G^{\prime \prime}(0)\right\}=\{-1,0\}$ for figure $4 \mathrm{~b}$ which ultimately unwind to the dual branch solution. In between the large- $\sigma$ primary and dual solution branches one finds what appears to be an endless set of multiple-solution branches. Indeed, Figure $4 \mathrm{~b}$ clearly shows this winding about $\{-1,0\}$, where we have calculated five upper branch solutions (I-V) and four lower branch solutions (iv-i). Comparisons of the large $\sigma$ asymptotics with the numerical results for the primary branch shear stress parameters are plotted in figure $4 \mathrm{c}$ in which excellent agreement is found for the two-term $G^{\prime \prime}(0)$ expression (6.7b) and good agreement is found for the one-term $F^{\prime \prime}(0)$ expression (6.7a). Velocity profiles for $F^{\prime}(\eta)$ and $G^{\prime}(\eta)$ at $\sigma=-1$ are plotted in figure $4 \mathrm{~d}$, and these show that as one moves towards the centre of the spiral, the $G^{\prime}(\eta)$ boundary layer becomes thicker and more oscillatory. Due to this observation, we find that in order to calculate more solution branches beyond those shown here, one would need to numerically integrate to values of $\eta_{\max }>20$; this in turn would require more accurate values of $F^{\prime \prime}(0)$ and $G^{\prime \prime}(0)$, which already are required to 14 significant figures in order to accurately calculate the branch iv solutions. This would require moving to quadruple precision. However, the results in figures 4a and 4b clearly exhibit the spiralling solution behaviors without the need to calculate additional solution branches.

The solution branches are delimited by turning points which are tabulated as left, $\sigma_{l}$, and right, $\sigma_{r}$, turning points in Table 1 and the existence of multiple solutions is shown in Table 2.

\begin{tabular}{|c|c|c|}
\hline Branch Transition & $\sigma_{l}$ & $\sigma_{r}$ \\
\hline \hline I - II & -1.4758 & - \\
\hline II - III & - & -0.8447 \\
\hline III - IV & -1.0498 & - \\
\hline IV - V & - & -0.9857 \\
\hline V - VI & -1.0045 & - \\
\hline iv - v & - & -0.9930 \\
\hline iii - iv & -1.0235 & - \\
\hline ii - iii & - & -0.9246 \\
\hline i - ii & -1.2224 & - \\
\hline
\end{tabular}

Table 1. Left turning points, $\sigma_{l}$, and right turning points, $\sigma_{r}$, showing the transitions between the computed solution branches. 


\begin{tabular}{|c|c|}
\hline No. of solutions & Range of $\sigma$ \\
\hline 0 & $\sigma<-1.4758$ \\
\hline 1 & $\sigma=-1.4758$ \\
\hline 2 & $-1.4758<\sigma<-1.2224$ \\
\hline 3 & $\sigma=-1.2224$ \\
\hline 4 & $-1.2224<\sigma<-1.0498$ \\
\hline 5 & $\sigma=-1.0498$ \\
\hline 6 & $-1.0498<\sigma<-1.0235$ \\
\hline 7 & $\sigma=-1.0235$ \\
\hline 8 & $-1.0235<\sigma<-1.0045$ \\
\hline 9 & $\sigma=-1.0045$ \\
\hline$>9$ & $-1.0045<\sigma<-0.9930$ \\
\hline 9 & $\sigma=-0.9930$ \\
\hline 8 & $-0.9930<\sigma<-0.9857$ \\
\hline 7 & $\sigma=-0.9857$ \\
\hline 6 & $-0.9857<\sigma<-0.9246$ \\
\hline 5 & $\sigma=-0.9246$ \\
\hline 4 & $-0.9246<\sigma<-0.8447$ \\
\hline 3 & $\sigma=-0.8447$ \\
\hline 2 & $\sigma>-0.8447$ \\
\hline
\end{tabular}

Table 2. Existence of multiple solutions as a function of $\sigma$.

\subsection{Stability of multiple solutions}

As in $\S 4.4$ we introduce the dimensionless time $\tau=$ at and include the unsteady acceleration in the Navier-Stokes equations and this leads to the coupled equations

$$
\begin{aligned}
& F^{\prime \prime \prime}+(F+G) F^{\prime \prime}-F^{2}-F_{\tau}^{\prime}+1=0 \\
& G^{\prime \prime \prime}+(F+G) G^{\prime \prime}-G^{2}-G_{\tau}^{\prime}+1=0
\end{aligned}
$$

which, upon substituting the ansatz given in (4.17) again leads exactly to the eigenvalue problem (4.18) with boundary conditions (4.19).

Results for the lowest eigenvalues $\lambda_{1}$ and those for $G_{1}^{\prime \prime}(0)$ as a function of $\sigma$ are plotted in figures $4 \mathrm{e}$ and $4 \mathrm{f}$, respectively. The results in figure $4 \mathrm{e}$ show that despite the interesting behavior of multiple-solution branches, it is in fact only the primary branch solutions which are stable, and all other branches are all unstable. However it is interesting to note that the dual and primary branch solutions spiral in to different eigenvalues $\lambda_{1}^{\text {lower }}$ and $\lambda_{1}^{\text {upper }}$ with $\lambda_{1}^{\text {upper }}>\lambda_{1}^{\text {lower }}$. 


\section{Discussion and Conclusion}

This paper provides a unified formulation for stagnation-point flow and linearly stretching plates wherein the two can occur separately, or in unison. After demonstrating the reduction of the unified formulation to known cases in the literature, three new problems were investigated: (1) a radially stretching plate below Hiemenz (1911) stagnation flow, (2) Crane (1970) stretching transverse to Hiemenz stagnation flow, and (3) Crane stretching beneath Homann (1936) stagnation flow. In each case numerical results were presented for the wall shear stresses as a function of the problem parameter, $\sigma$, and for problems (1) and (2), dual solutions were found to exist for $\sigma_{t}<\sigma$ where $\sigma_{t}$ is a turning point, and no solutions were found for $\sigma<\sigma_{t}$. Stability results show in both cases that the primary (upper) solution branches are stable while the dual (lower) solution branches are unstable. Asymptotic results are also presented for the stable solutions.

Results for problem (3) display much more interesting behavior, wherein the wall shear stresses reveal multi-branch spiralling behaviors. Along these spirals the velocity profiles $F^{\prime}(\eta)$ and $G^{\prime}(\eta)$ become thicker, and more oscillatory in the case of $G^{\prime}(\eta)$ as the centre of the spiral is approached. Despite this feature, it was again found that only the primary (upper) solution branch is stable, while all other multiple-solution branches are unstable.

As such interesting behavior is evident in the single-parameter problems analyzed here, the next stage is to consider multi-parameter problems for bi-axial stretching surfaces below stagnation-point flows, be they of Hiemenz, Homann or Howarth type. We leave the analysis of these more complicated flows to a future study. 


\section{References}

Crane, L.J., 1970. Flow past a stretching sheet. Z. Angew. Math. Phys. (ZAMP) 21, 645-647.

Davey, A., 1961. Boundary-layer flow at a saddle point of attachment. J. Fluid Mech. 10, 593-610.

Hiemenz, K., 1911. Die Grenzschicht an einem in den gleichförmuningen Flussigkeitsstrom eingetauchten geraded Kreiszylinder. Dinglers Poly. J. 326, 321-410.

Homann, F., 1936. Der Einfluss grosser Zähigkeit bei der Strömung um den Zylinder und um die Kugel. Z. angew. Math. Mech. (ZAMM) 16, 153-164.

Howarth, L., 1951. The boundary layer in three dimensional flow. Part II. The flow near a stagnation point, Phil. Mag. 42, 1433-1440.

Mahapatra, T.R. and Gupta, A.S. 2003. Stagnation-point flow towards a stretching surface. Can. J. Chem. Engr. 81, 258-263.

Mahapatra, T.R. and Gupta, A.S. 2002. Heat transfer in stagnation-point flow towards a stretching sheet. Heat Mass Transfer 38, 517-521.

Merkin, J.H. 1985. On dual solutions occurring in mixed convection in a porous medium. J. Engng. Math. 20, 171-179.

Press, W.H., Flannery, B.P., Teukolsky, S.A. and Vetterling, W.T., 1989. Numerical Recipes. Cambridge University Press, Cambridge.

Schlichting, H., 1960. Boundary layer theory. McGraw-Hill, New York.

Schmid, P.J. and Henningson, D.S., 2012. Stability and Transition in Shear Flows. Springer, New York.

Wang, C.Y., 1984. The three-dimensional flow due to a stretching flat surface. Phys. Fluids 27, 1915-1917.

Wang, C.Y., 2008a. Stagnation flow towards a shrinking sheet. Int. J. Non-linear Mech. 43, 377-382.

Wang, C.Y., 2008b. Similarity stagnation point solutions of the Navier-Stokes equations review and extension. Eur. J. Mech. B/Fluids 27, 678-683. 
Weidman, P.D. and Ishak, A., 2015. Multiple solutions of two-dimensional and threedimensional flows induced by a stretching flat surface. Commun. Nonlinear Sci. Numer. Simulat. 25, 1-9.

Weidman, P.D. and Sprague, M., 2015. Steady and unsteady modelling of the float height of a rotating air hockey disk. J. Fluid Mech. 778, 39-59. 


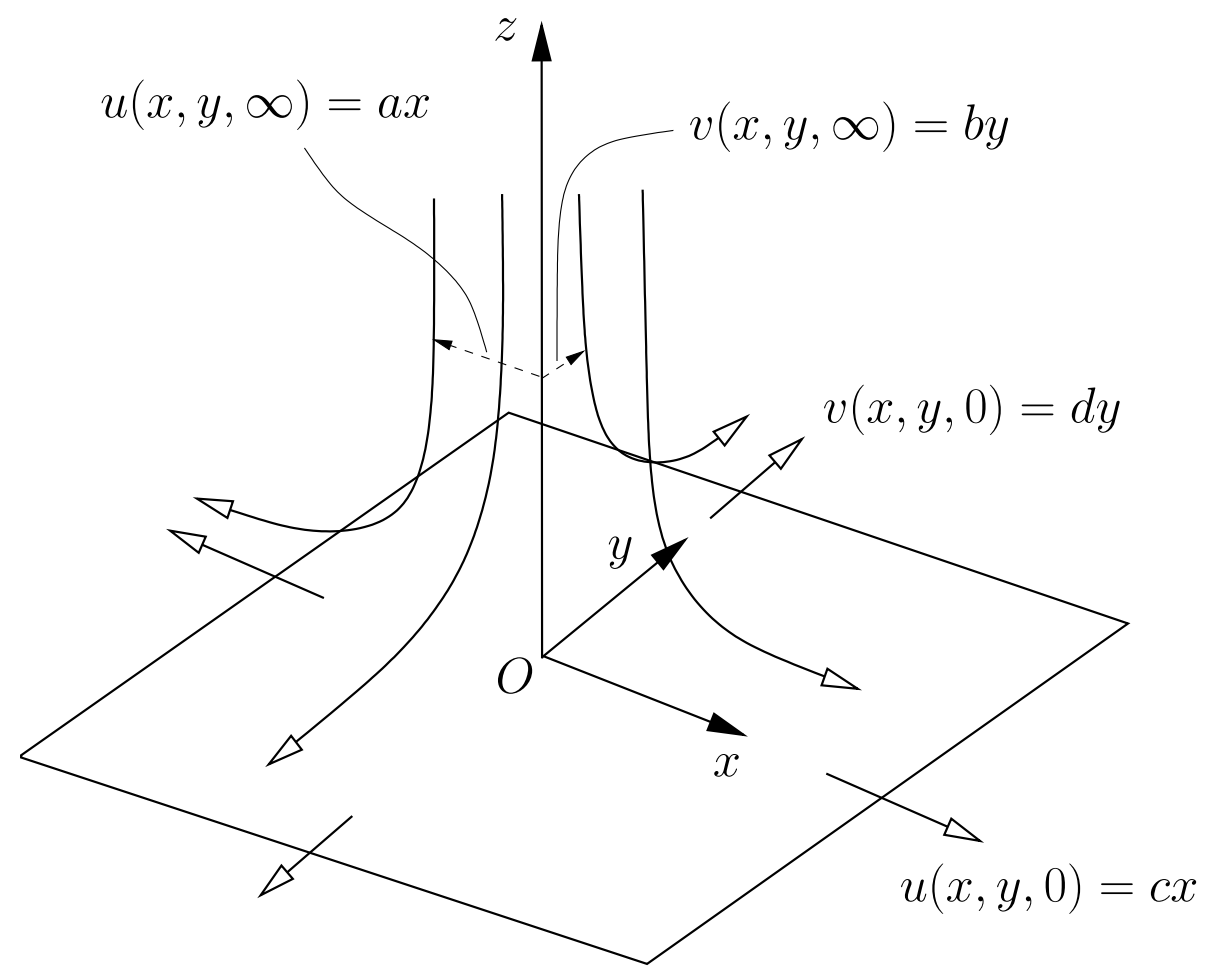

Figure 1. Schematic diagram of a stagnation point flow above a bi-axial stretching surface. 

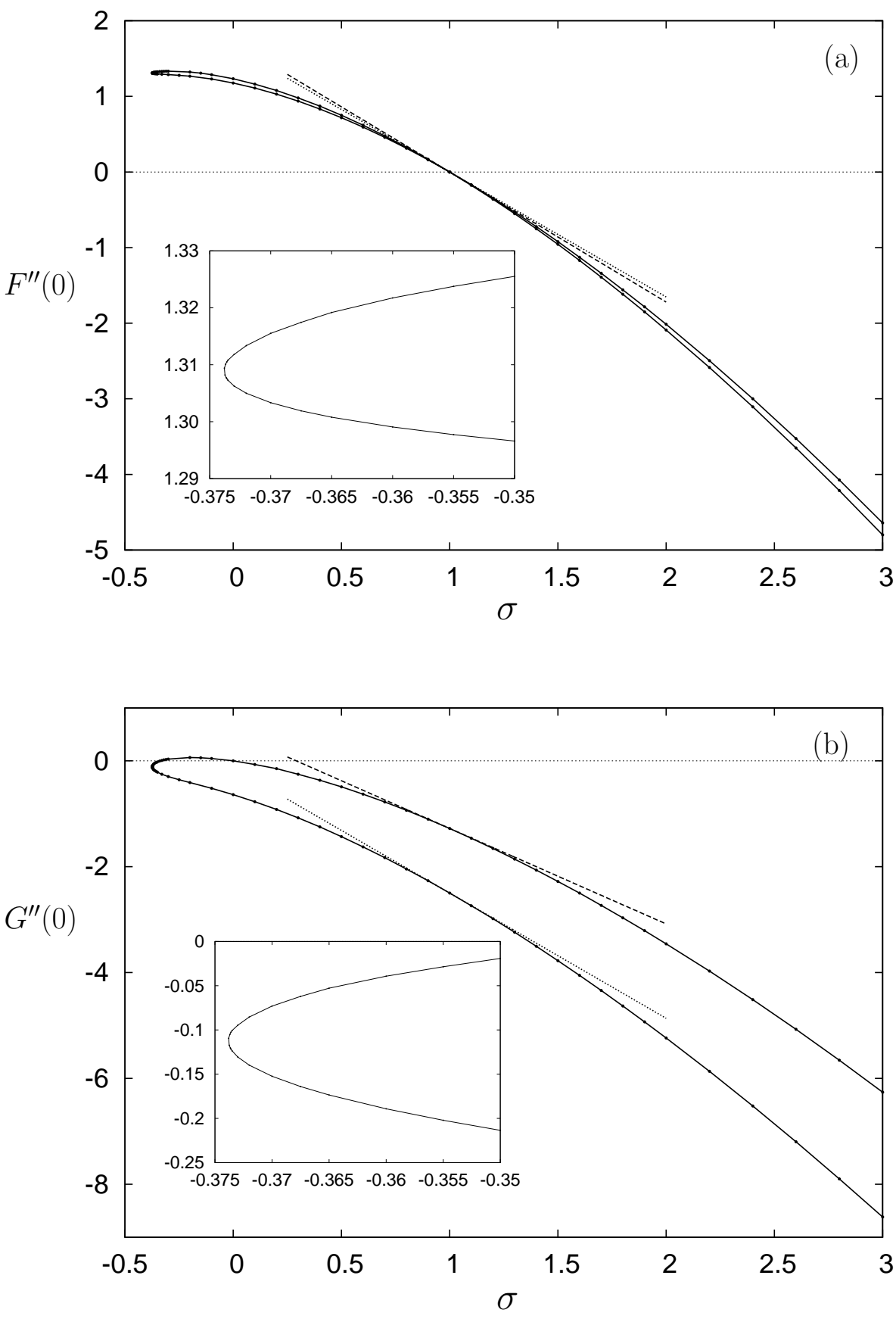

Figure 2. Wall shear stress parameters for radial stretching beneath Hiemenz stagnation-point flow. (a) Longitudinal stress parameter $F^{\prime \prime}(0)$ as a function of $\sigma$ with inset showing details near the turning point $\sigma_{t}=-0.3737$; (b) Transverse stress parameter $G^{\prime \prime}(0)$ as a function of $\sigma$ with inset showing details near the turning point $\sigma_{t}$. The dashed and dotted lines are the local behaviors around $\sigma=1$ of the primary and dual solution branches, respectively. 


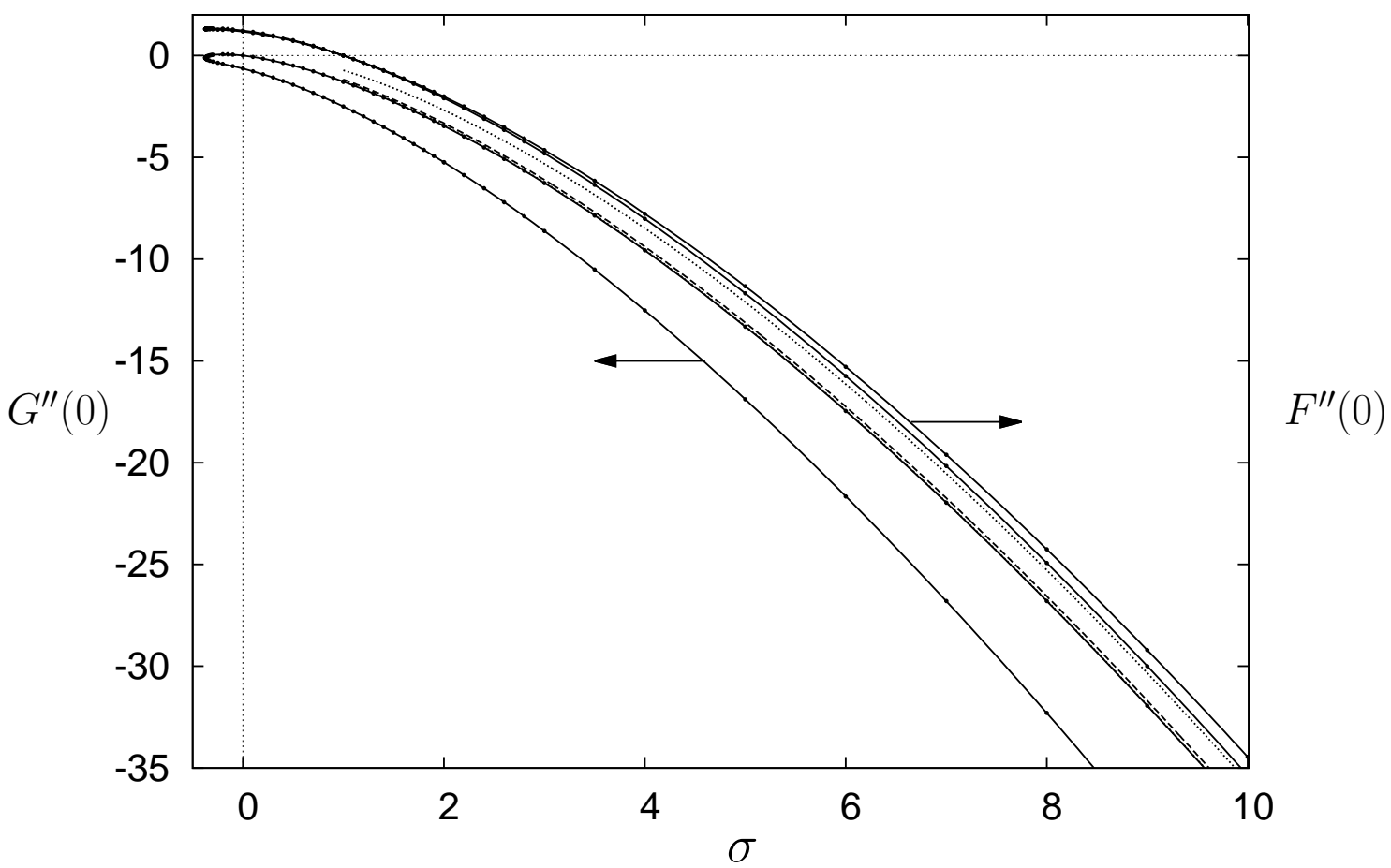

Figure 2c. Comparison of large- $\sigma$ asymptotic behaviors for the primary branch longitudinal and transverse wall shear stresses with numerical results shown a solid lines. The values of $F^{\prime \prime}(0)$ are on the left ordinate and the values of $G^{\prime \prime}(0)$ are on the right ordinate. The dashed lines are the one-term asymptotic behavior for both $F^{\prime \prime}(0)$ and $G^{\prime \prime}(0)$. The dotted line for the two-term asymptotic behavior of $G^{\prime \prime}(0)$ precisely overlays the primary solution branch. 

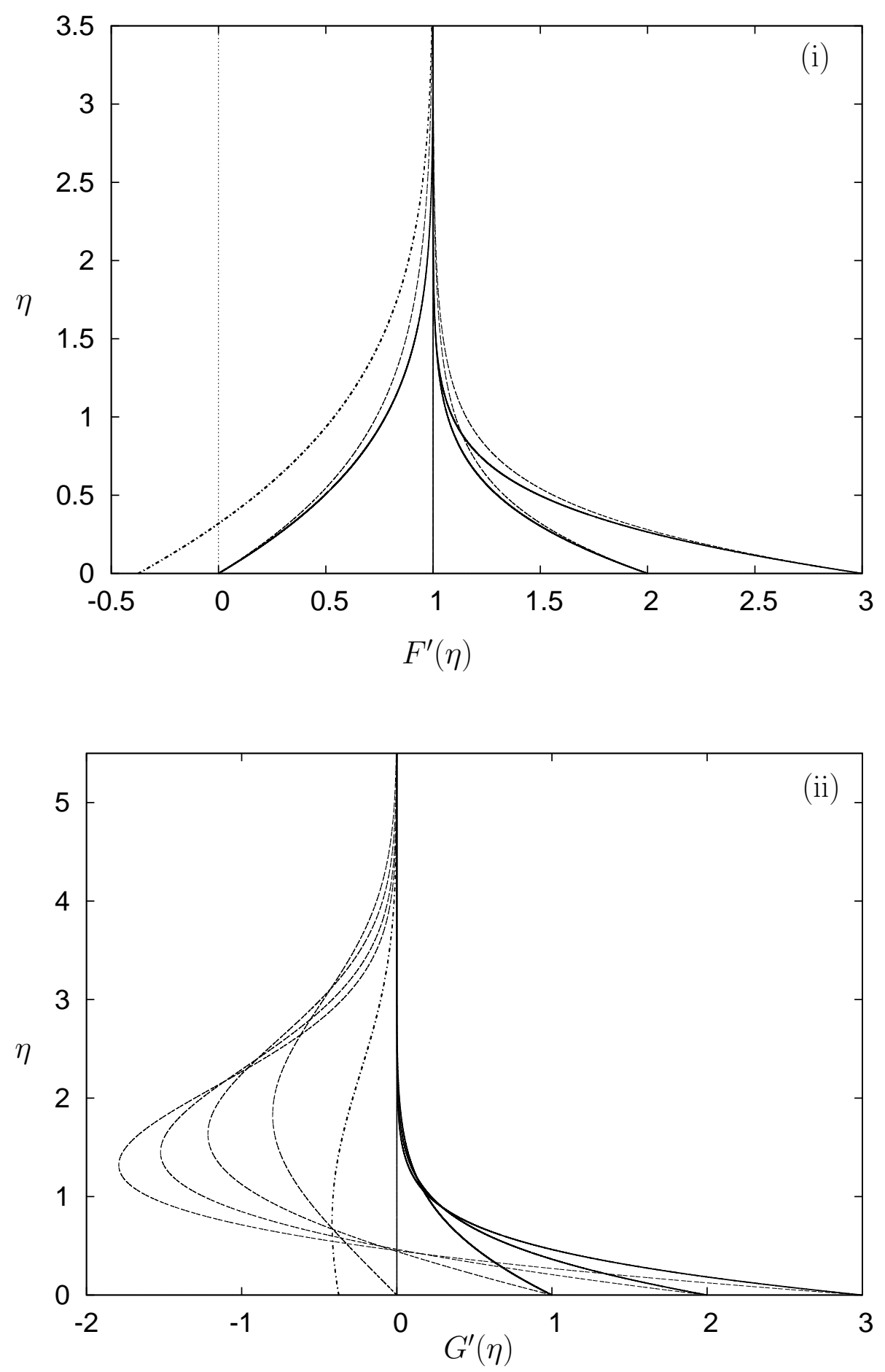

Figure 2d. Longitudinal and transverse velocities displayed at the turning point $\sigma_{t}=-0.3737$ and at $\sigma=0,1,2,3$; (i) longitudinal velocities $F^{\prime}(\eta)$ and (ii) transverse velocities $G^{\prime}(\eta)$. In each case the profiles along the primary branch are shown as solid lines, those along the dual branch are shown as dashed lines, and profiles at the turning point are shown as dash-dot-dash lines. 


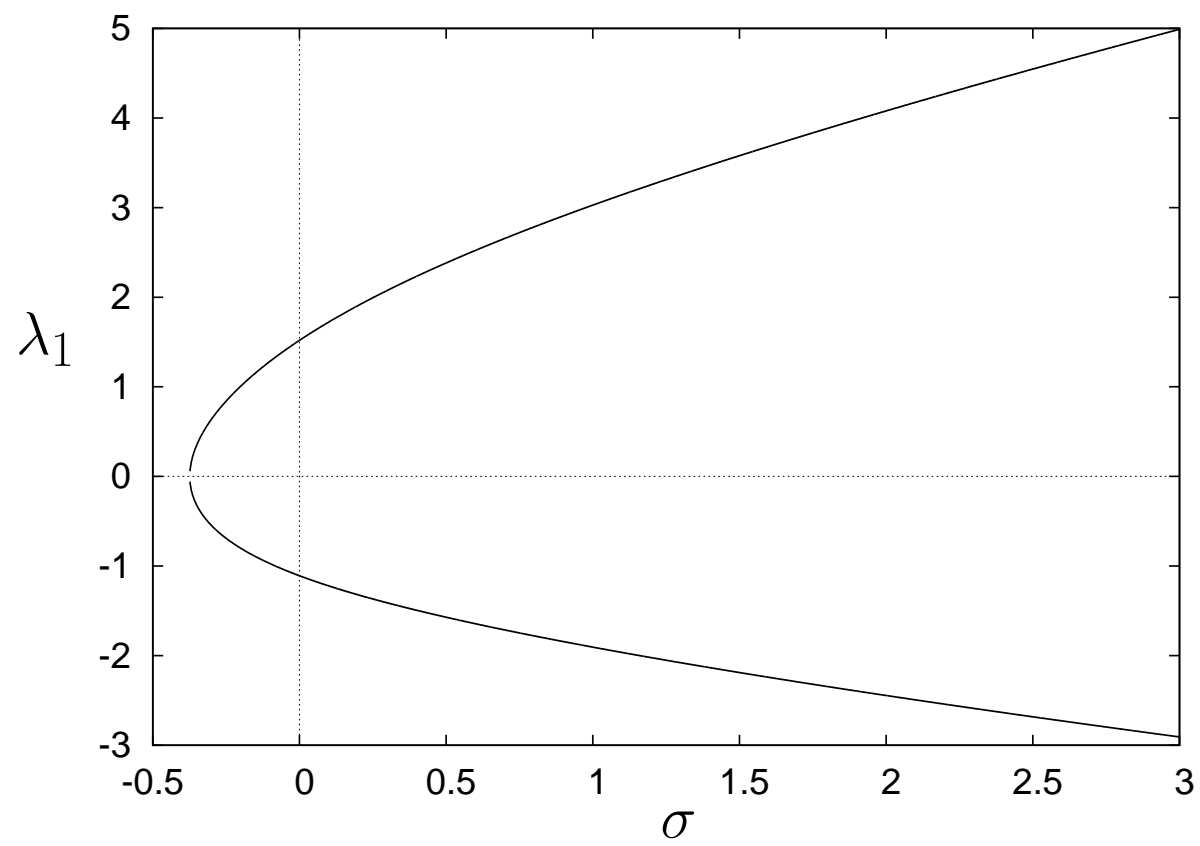

Figure 2e. Smallest eigenvalue $\lambda_{1}$ as a function of $\sigma$. The positive eigenvalues on the primary (upper) branch denote stable flow and the negative eigenvalues on the dual (lower) solution branch denote unstable flow.

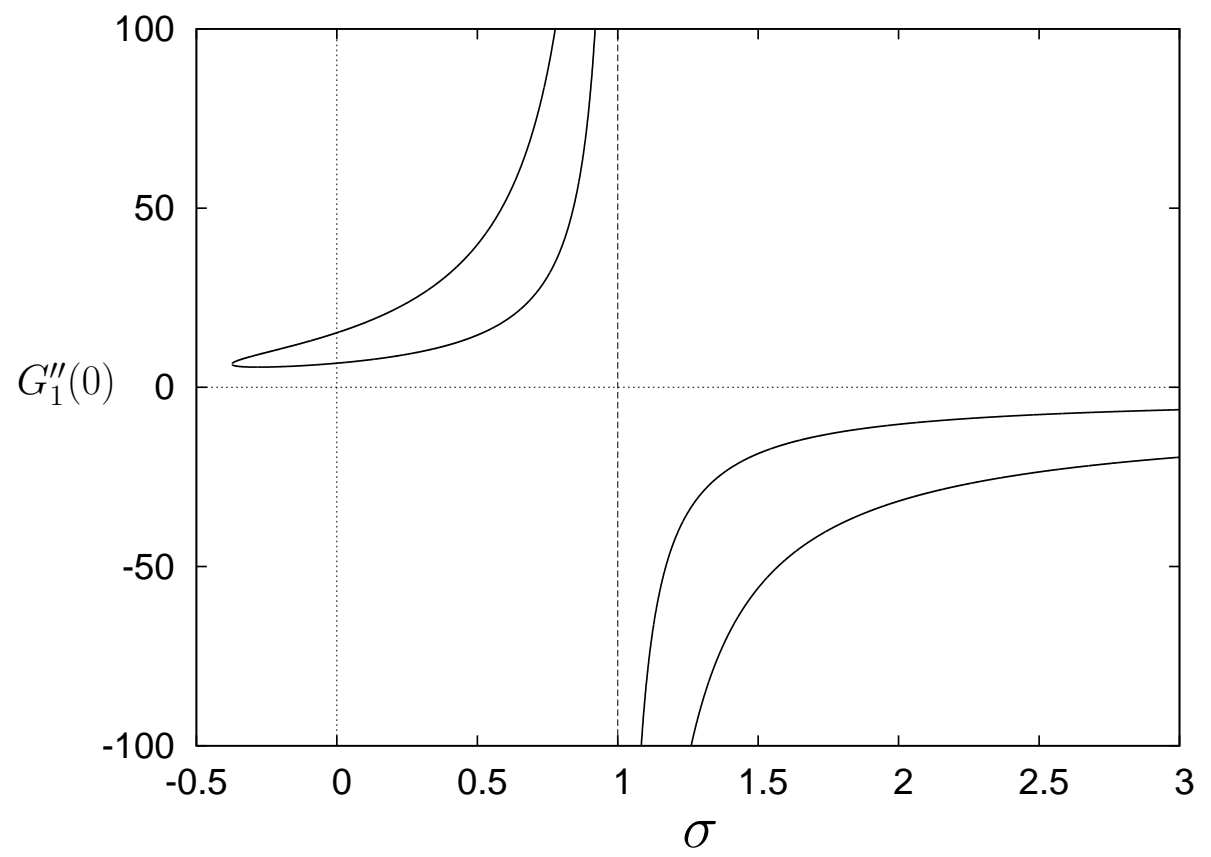

Figure 2f. Shear stress $G_{1}^{\prime \prime}(0)$ for the eigenfunction as a function of $\sigma$ for calculation of the lowest eigenvalue. The dashed line denotes a vertical asymptote. 

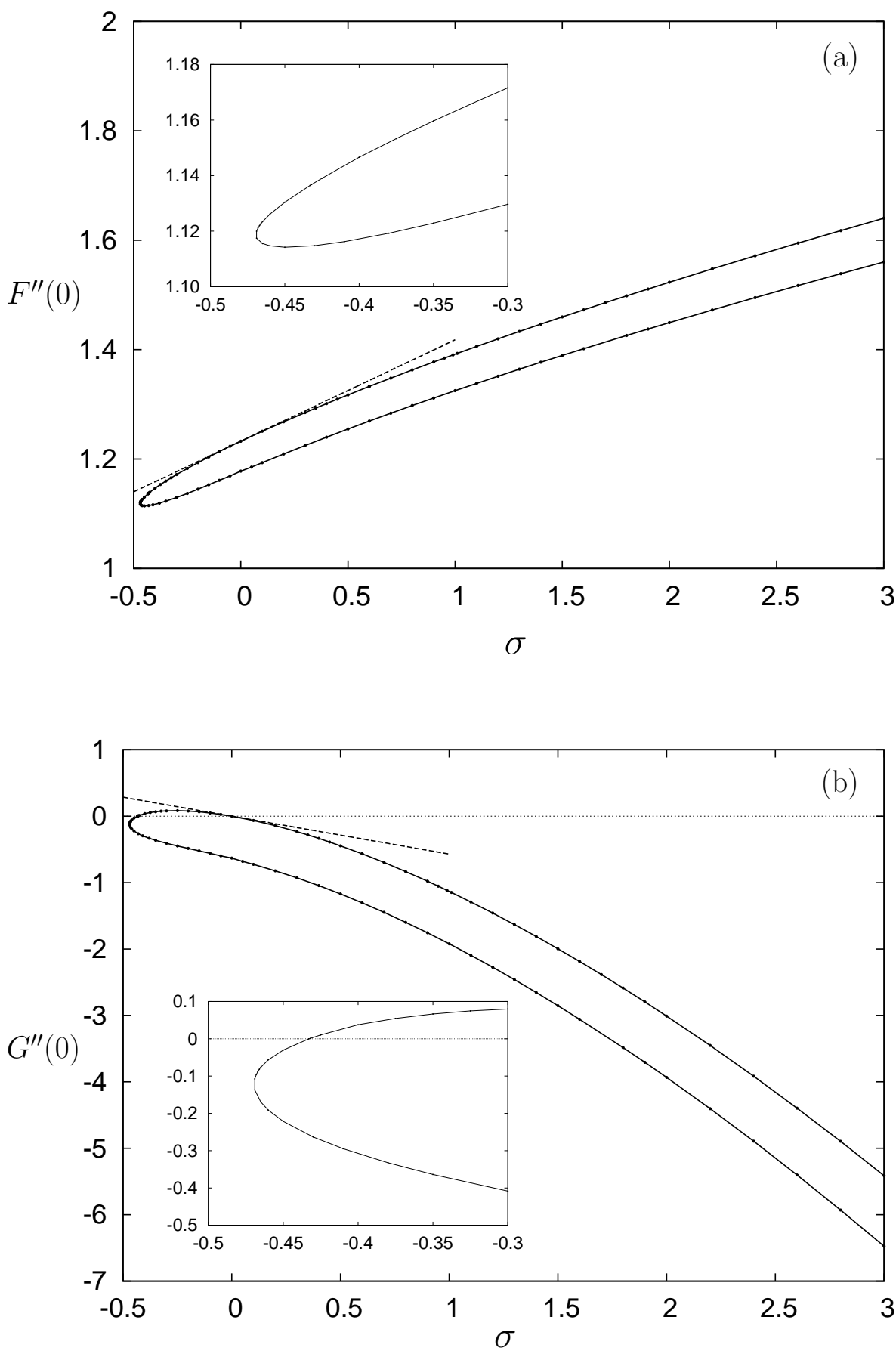

Figure 3. Wall shear stress parameters for Crane stretching transverse to Hiemenz stagnation-point flow. (a) Longitudinal stress parameter $F^{\prime \prime}(0)$ as a function of $\sigma$ with inset showing details near the turning point $\sigma_{t}=-0.4690$; (b) Transverse stress parameter $G^{\prime \prime}(0)$ as a function of $\sigma$ with inset showing details near the turning point $\sigma_{t}$. The dashed lines are the local behaviors around $\sigma=1$ of the primary solution branch. 


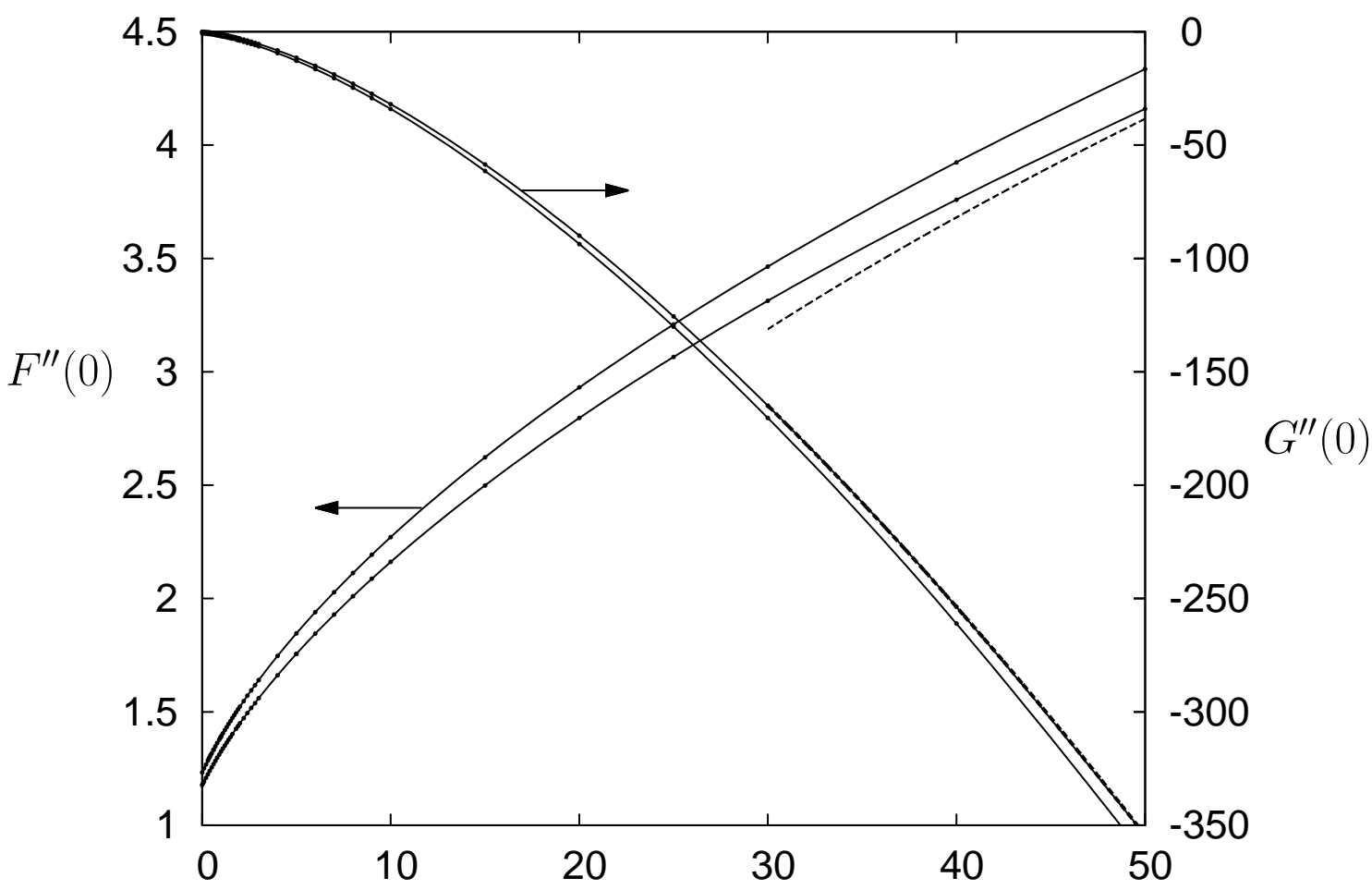

$\sigma$

Figure 3c. Comparison of large- $\sigma$ asymptotic behaviors for the primary branch longitudinal and transverse wall shear stresses with numerical results shown as solid lines. The dashed line is the one-term asymptotic behavior for both $F^{\prime \prime}(0)$ and $G^{\prime \prime}(0)$ and the dotted lines are the two-term asymptotic behaviors as reported in Eq. (6.8). The one-term result for $F^{\prime \prime}(0)$ is seen to approach the primary (upper) branch at large $\sigma$ while the two-term result for $G^{\prime \prime}(0)$ precisely overlays the primary (upper) solution branch. 

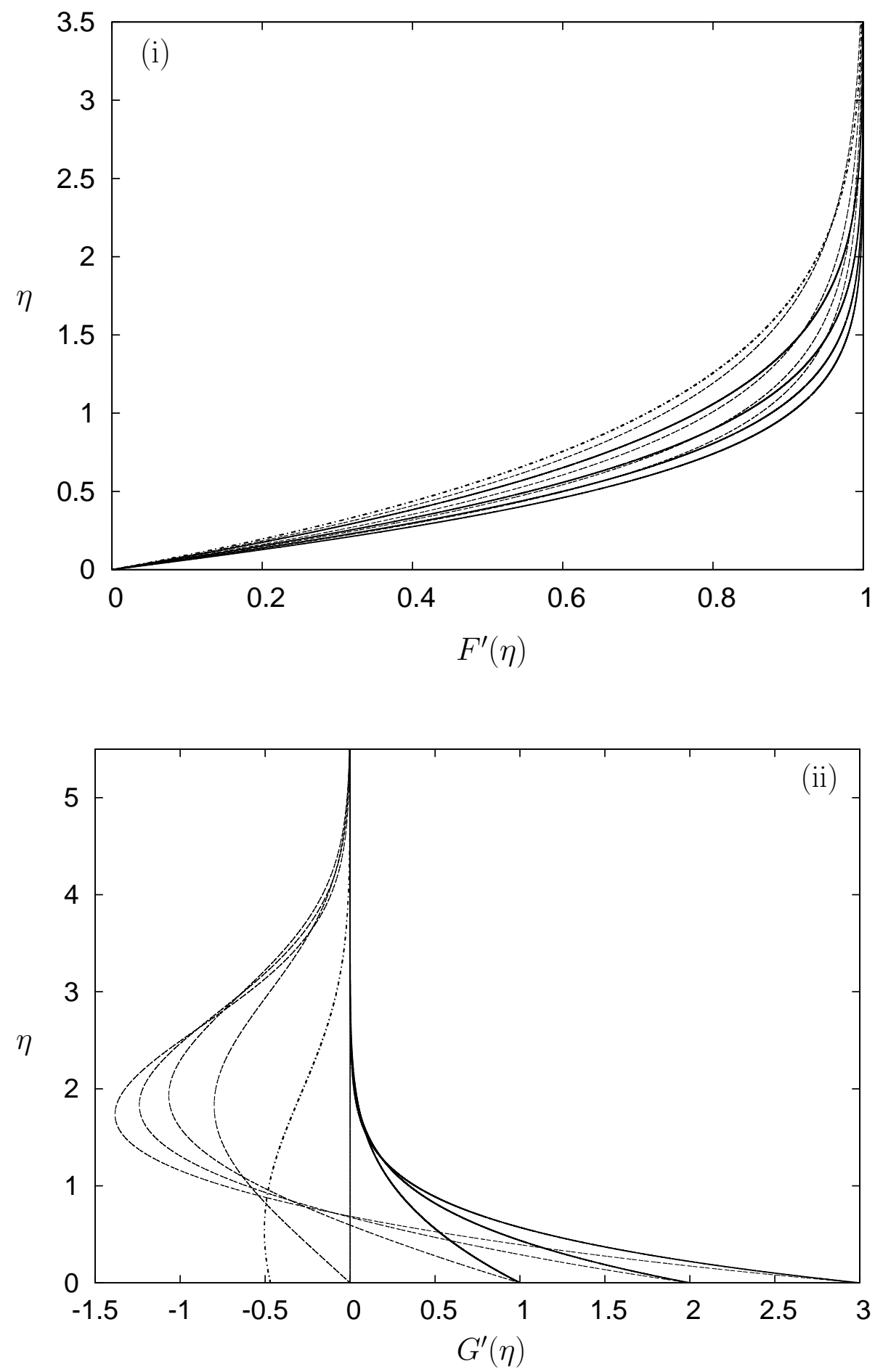

Figure 3d. Longitudinal velocities and transverse velocities displayed at the turning point $\sigma_{t}=-0.4690$ and at $\sigma=0,1,2,3$; (i) longitudinal velocities $F^{\prime}(\eta)$ and (ii) transverse velocities $G^{\prime}(\eta)$. In each case the profiles along the primary branch are shown as solid lines, those along the dual branch are shown as dashed lines, and profiles at the turning point are shown as dash-dot-dash lines. 


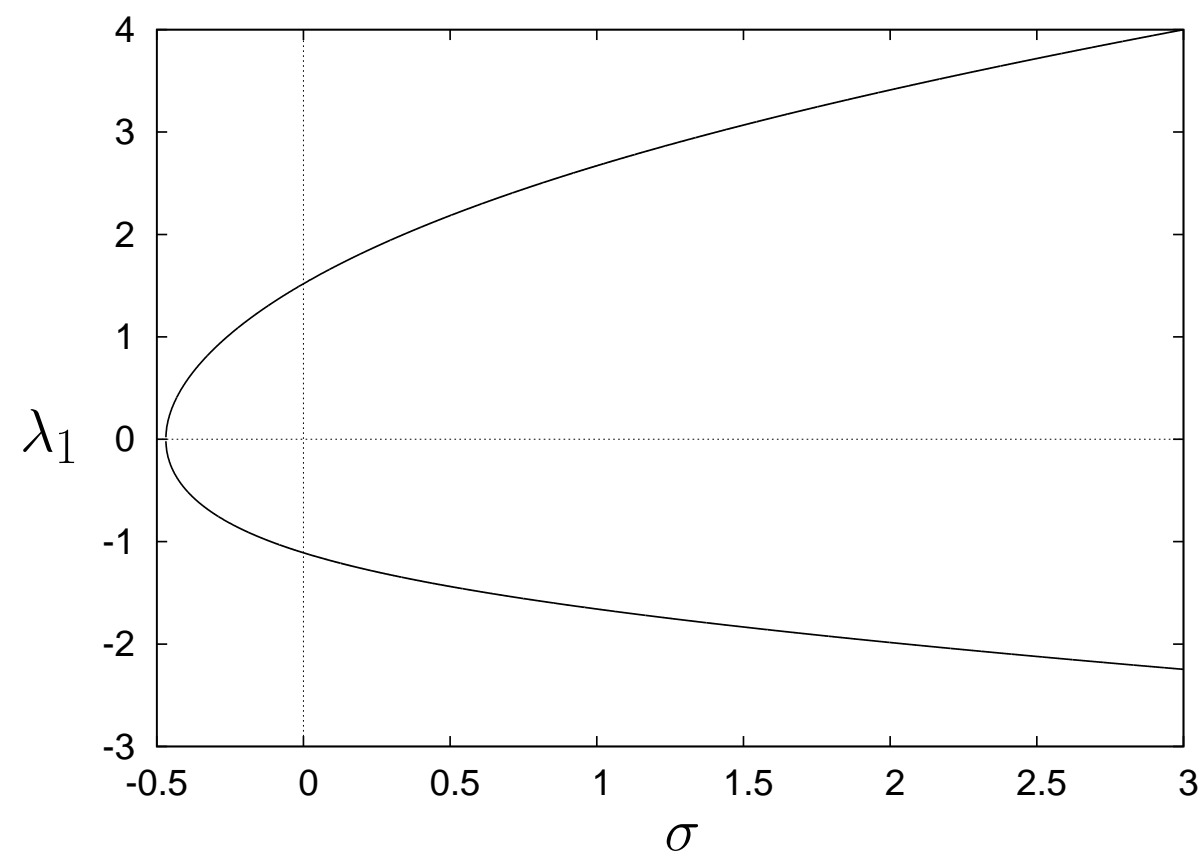

Figure 3e. Smallest eigenvalue $\lambda_{1}$ as a function of $\sigma$. The positive eigenvalues on the primary (upper) solution branch denote stable flow whilst the negative eigenvalues on the dual (lower) solution branch denote unstable flow.

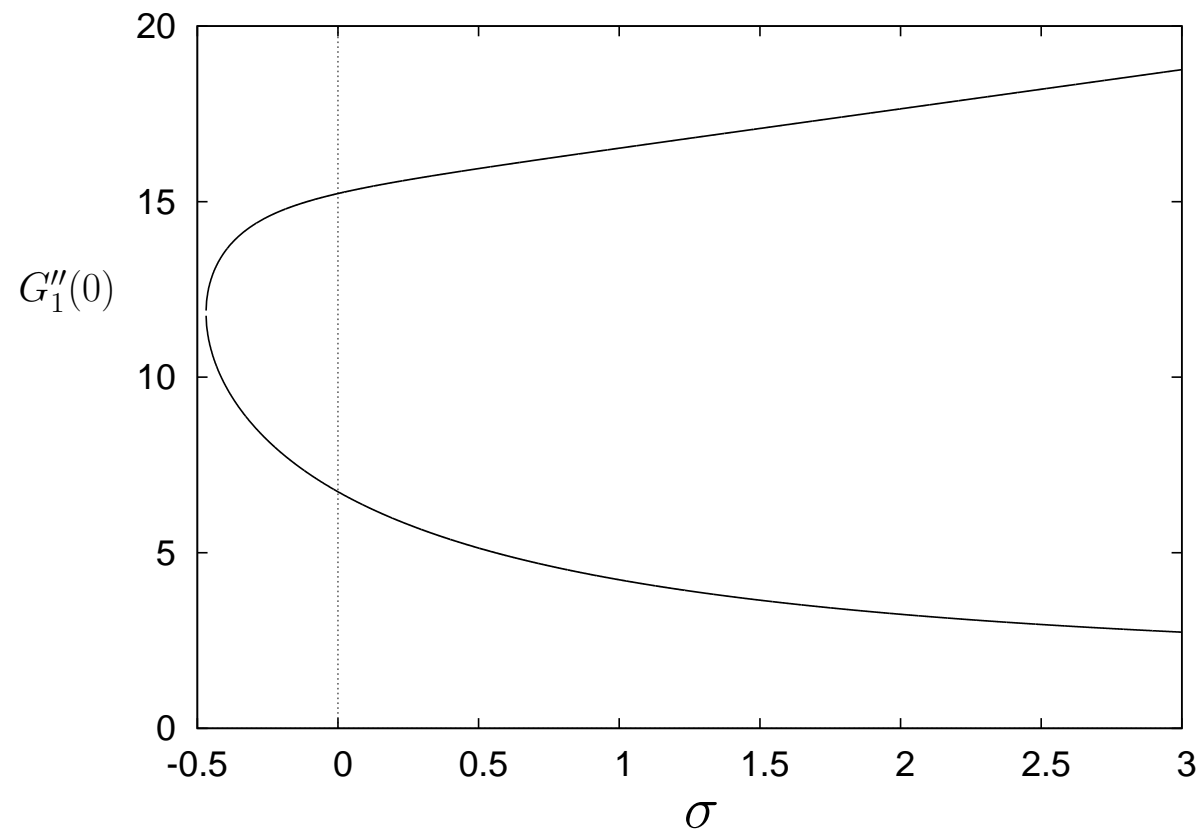

Figure 3f. Shear stress $G_{1}^{\prime \prime}(0)$ for the eigenfunction as a function of $\sigma$ for calculation of the lowest eigenvalues shown in figure $3 \mathrm{e}$. 

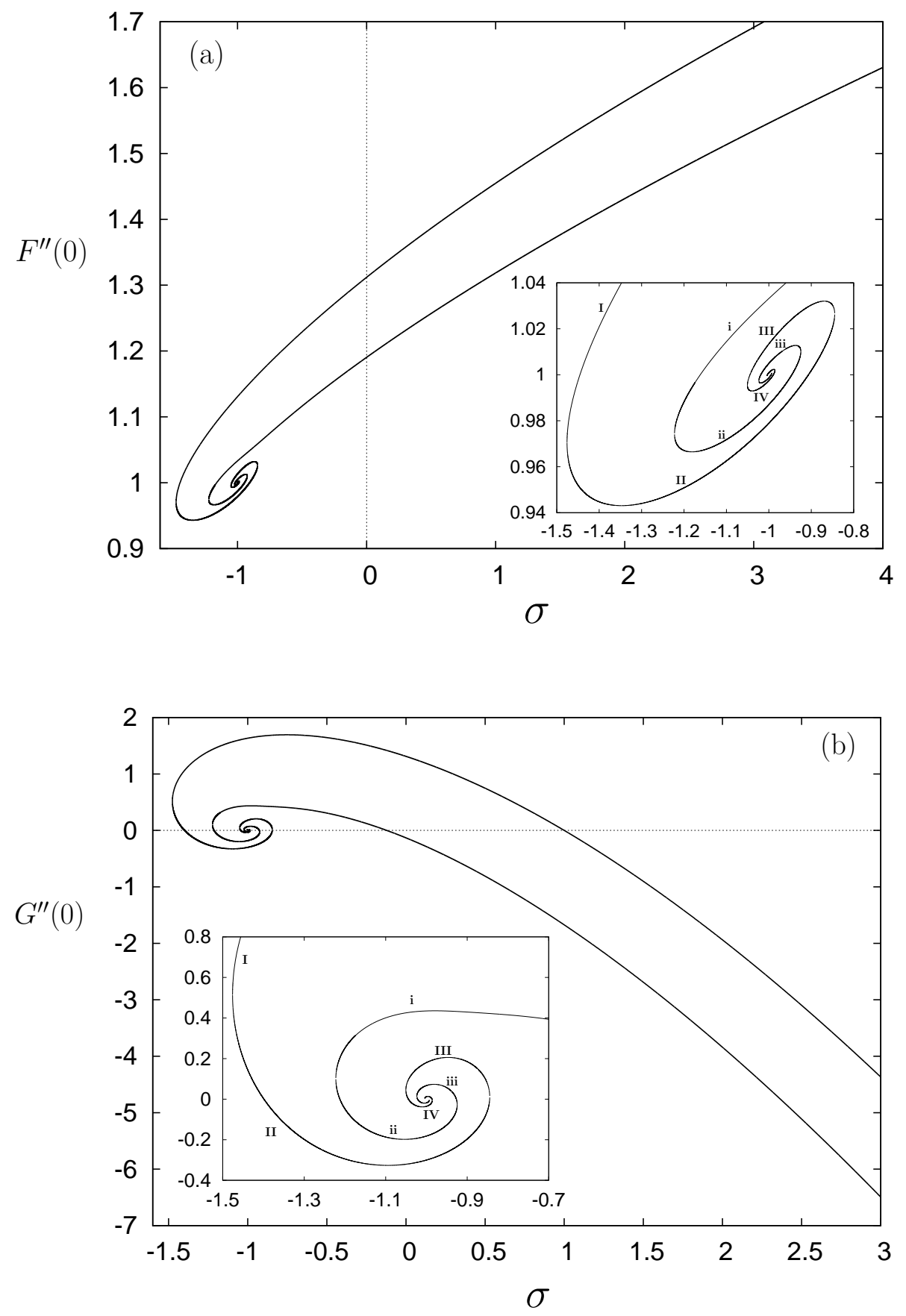

Figure 4. Wall shear stress parameters for Crane stretching beneath Homann stagnation-point flow. (a) Longitudinal stress parameter $F^{\prime \prime}(0)$ and (b) transverse stress parameter $G^{\prime \prime}(0)$ as a function of $\sigma$. The insets show the solution branches winding in spiral fashion towards the points $\left\{\sigma, F^{\prime \prime}(0)\right\}=\{-1,1\}$ and $\left\{\sigma, G^{\prime \prime}(0)\right\}=\{-1,0\}$. In both cases nine solution branches are shown, five upper branch solutions (I-V) and four lower branch solutions (iv-i). 


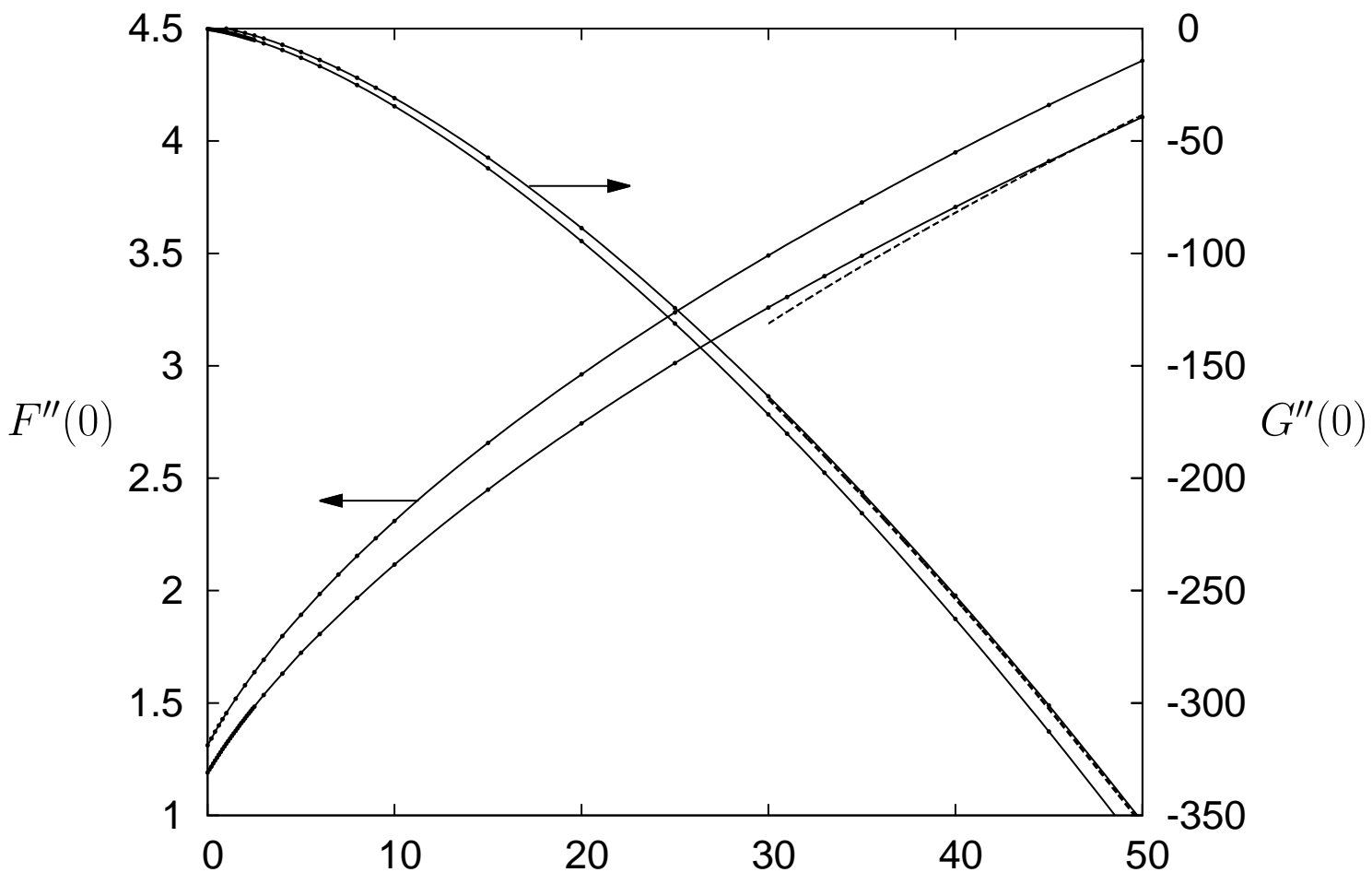

Figure 4c. Comparison of large- $\sigma$ asymptotic behaviors for the primary (upper) solution longitudinal and transverse wall shear stresses with numerical results. The dashed lines are the asymptotic behaviors of $F^{\prime \prime}(0)$ and $G^{\prime \prime}(0)$ given in Eq. (6.7) and the solid lines are the numerical results. 

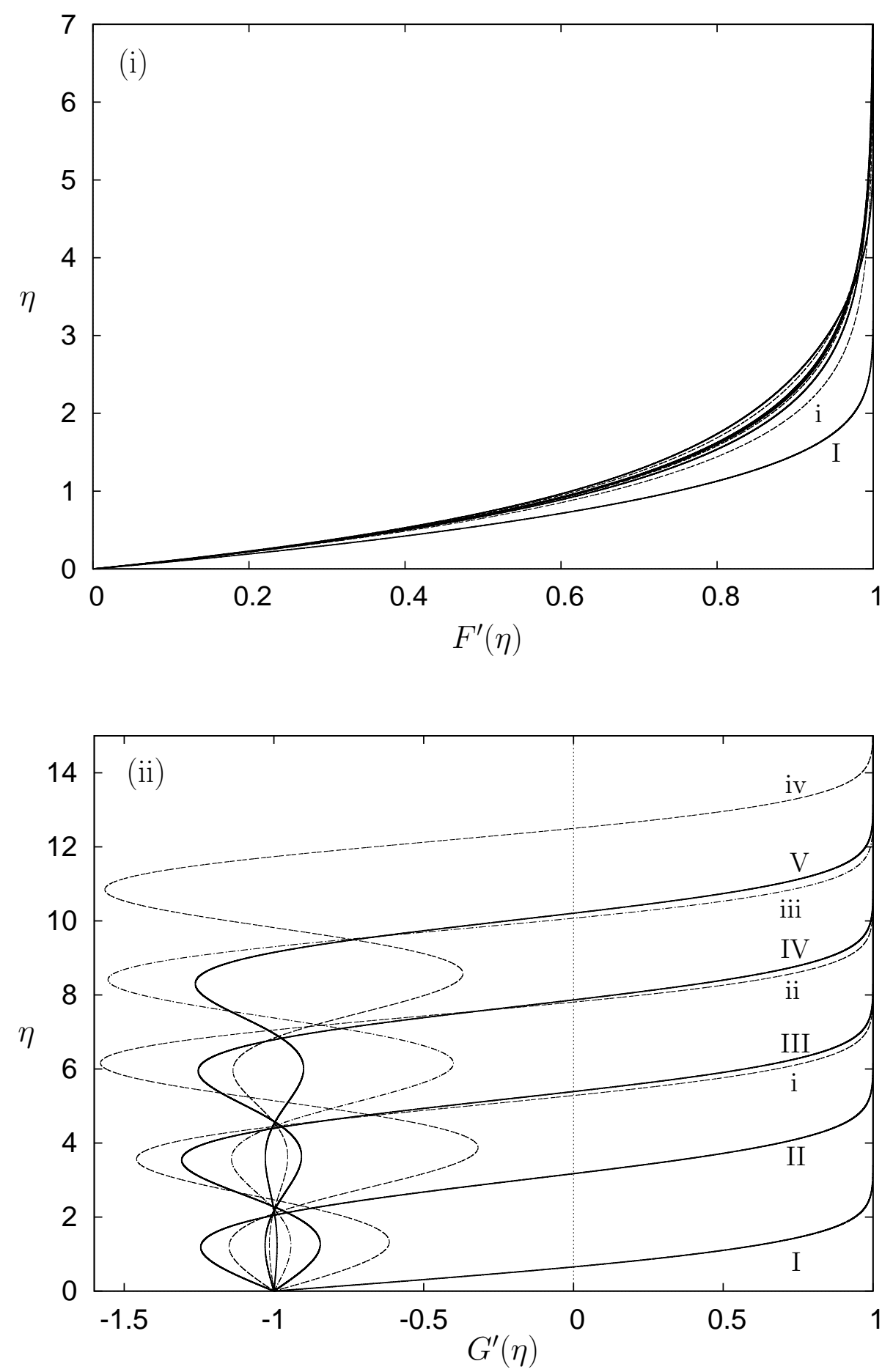

Figure 4d. Longitudinal and transverse velocities cut through the point $\sigma=-1$ where multiple solutions exist; (i) longitudinal velocities $F^{\prime}(\eta)$ for the first five computed upper branch solutions are shown as solid lines, while the first four lower branch solutions are shown by dashed lines; (ii) the transverse velocities $G^{\prime}(\eta)$ are labeled similarly. 


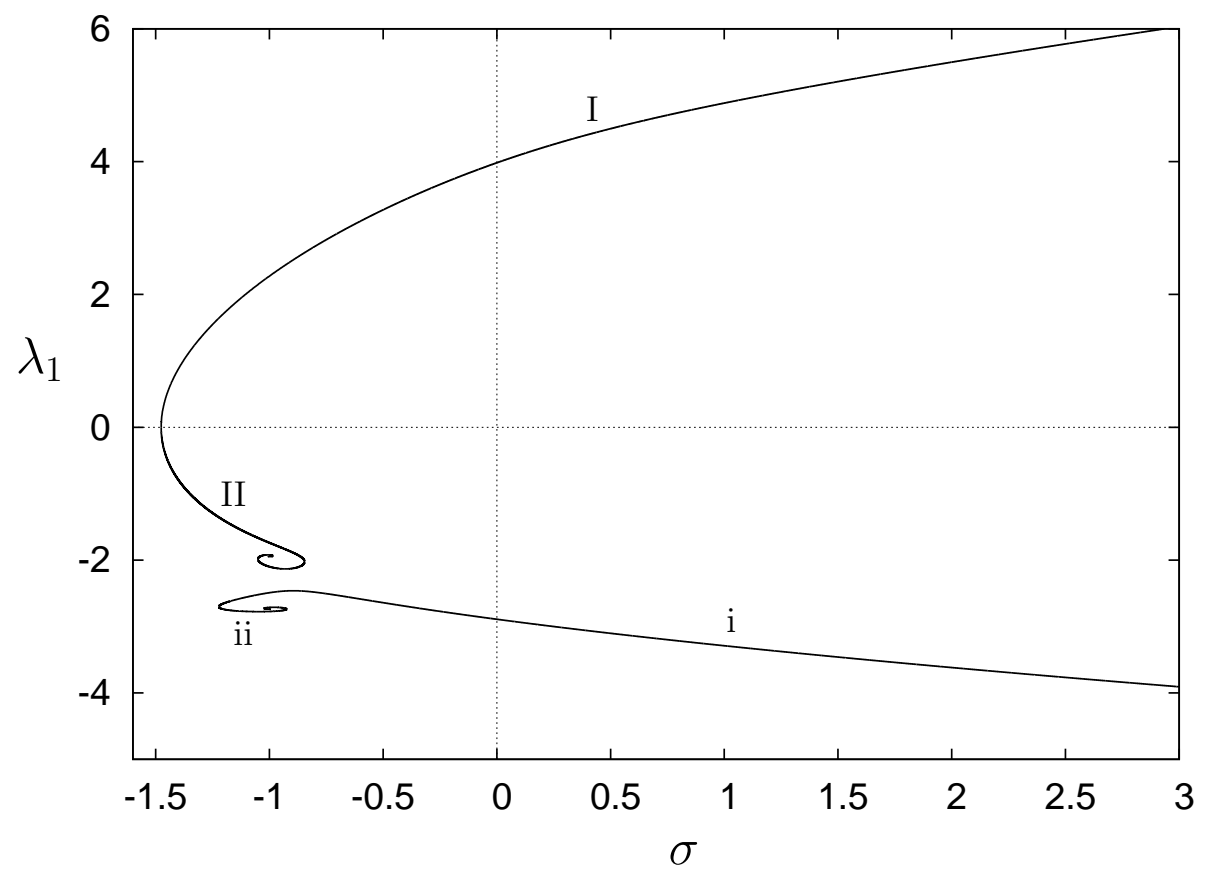

Figure 4e. Smallest eigenvalue $\lambda_{1}$ as a function of $\sigma$. The positive eigenvalues on the uppermost branch denote stable flow whilst the negative eigenvalues for all other branches denote unstable flow.

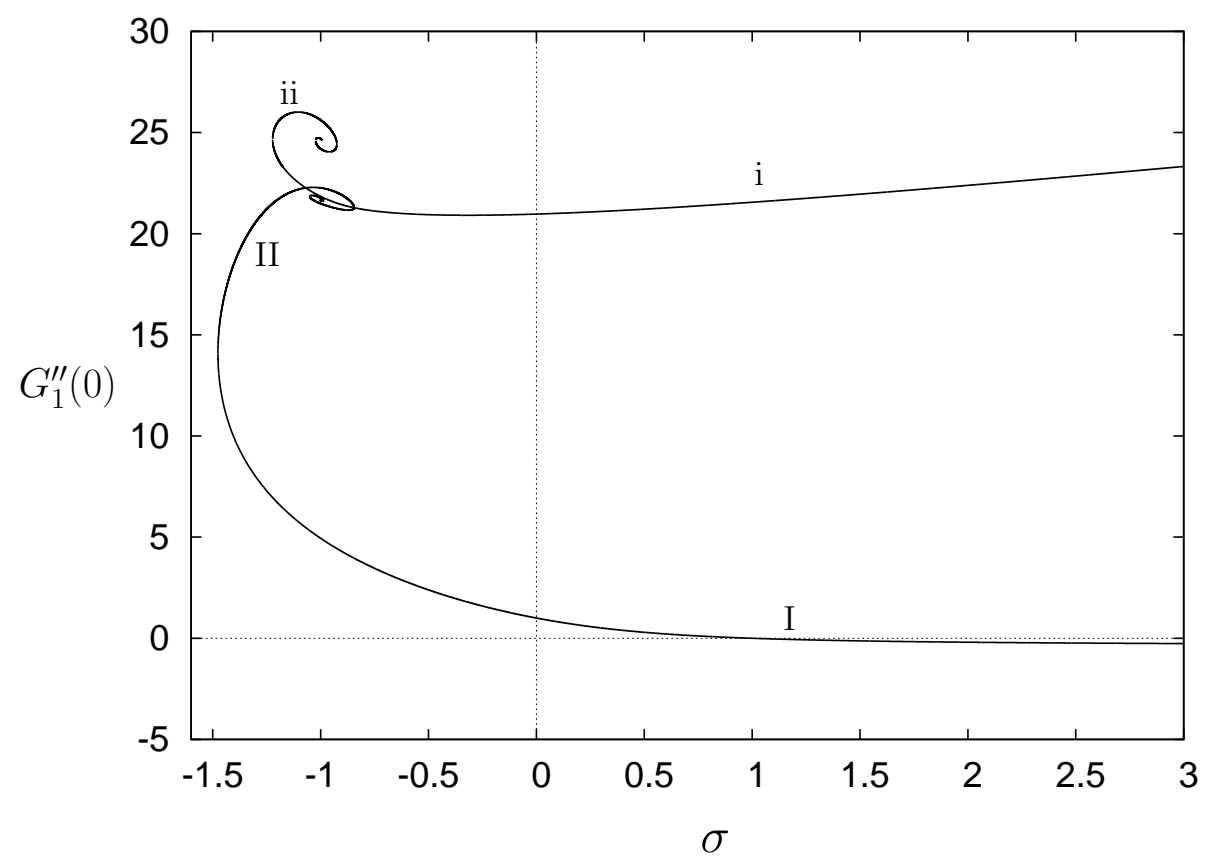

Figure 4f. Shear stress $G_{1}^{\prime \prime}(0)$ for the eigenfunction as a function of $\sigma$ for calculation of the lowest eigenvalues shown in figure $4 \mathrm{e}$. 